\title{
$n 0.342$
}

\section{MULTIPLICATION SEMIGROUPS}

Vijaya, L. Mannepalli

\section{A Dissertation}

Submitted to the Graduate School of Bowling Green State University in partial fulfillment of the requirements for the degree of

\author{
DOCTOR OF PHILOSOPHY
}

Deeember 1975

Approved by Doctoral Committee M. Saty anarayan Advisor Department of Mathematics
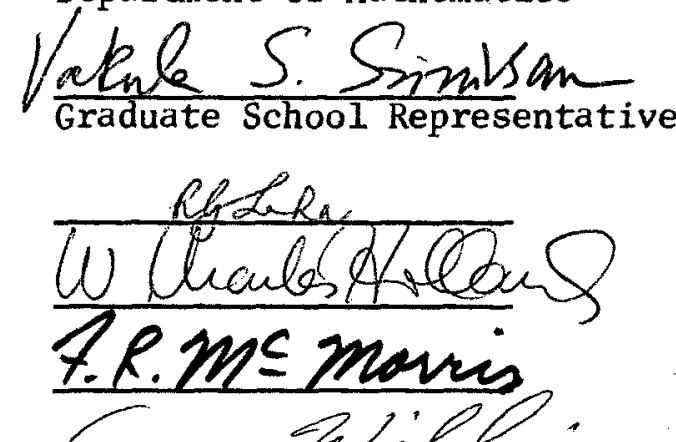
The theory of regular semigroups is of paramount importance in the general framework of the structure of semigroups. During the last few years the general construction of regular semigroups has gained the attention of Clifford, Grillet, Namboodripad etc.

In this work a new property called multiplication property enjoyed by many classes of semigroups including regular semigroups, has been introduced. It has been shown that this property plays an important role in distinguishing regular semigroups among the bigger class of semisimple semigroups. Construction of left cancellative semigroups satisfying multiplication property has been obtained. It is proved that the multiplication property separates commutative principal ideal semigroups among the commutative noetherian semigroups. Conditions have been obtained for a commutative noetherian semigroup with multiplication property to be a regular semigroup. Furthermore in the commutative case the structure of multiplication semigroups (semigroups satisfying multiplication property) has been determined in the noetherian case and the finite-dimensional case. 


\section{ACKNOWLEDGEMENT}

I would like to thank my major adviser, Dr. M. Satyanarayana, for his valuable guidance and constant encouragement during the preparation of this Dissertation. 
CONTENTS

Page

INTRODUCTION $\ldots \ldots \ldots \ldots \ldots \ldots \ldots \ldots \ldots \ldots \ldots \ldots \ldots \ldots \ldots, 1$

1. MULTIPLICATION SEMIGROUPS $\ldots \ldots \ldots \ldots \ldots \ldots \ldots \ldots \ldots \ldots \ldots \ldots, 3$

1.1 Basic Definitions $\ldots \ldots \ldots \ldots \ldots \ldots \ldots \ldots \ldots \ldots \ldots \ldots \ldots$

1.2 General Properties ............................... 5

1.3 Properties of Multiplication Semigroups $\ldots \ldots \ldots \ldots \ldots \ldots . .6$

1.4 Semisimple Semigroups $\ldots \ldots \ldots \ldots \ldots \ldots \ldots \ldots \ldots \ldots \ldots \ldots \ldots \ldots$

1.5 Left-cancellative Semigroups $\ldots \ldots \ldots \ldots \ldots \ldots \ldots \ldots \ldots \ldots . \ldots \ldots$

2. COMMUTATIVE MULTIPLICATION SEMIGROUPS $\ldots \ldots \ldots \ldots \ldots \ldots \ldots \ldots, 21$

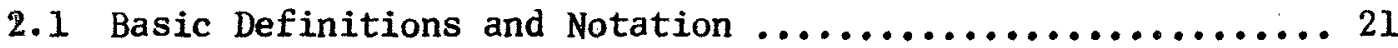

2.2 General Properties of Commutative Semigroups $\ldots \ldots \ldots \ldots \ldots . \ldots 24$

2.3 Properties of Commutative Multiplic ation Semigroups ...... 31

2.4 Commutative Noetherian Semigroups $\ldots \ldots \ldots \ldots \ldots \ldots \ldots \ldots . \ldots 4$

2.5 Commutative Finite-dimensional Semigroups $\ldots \ldots \ldots \ldots \ldots \ldots 48$

2.6 Some Additional Characterizations of Commutative Multiplication Semigroups $\ldots \ldots \ldots \ldots \ldots \ldots \ldots \ldots \ldots \ldots, 53$

2.7 Commutative Chained Semigroups $\ldots \ldots \ldots \ldots \ldots \ldots \ldots \ldots \ldots \ldots$

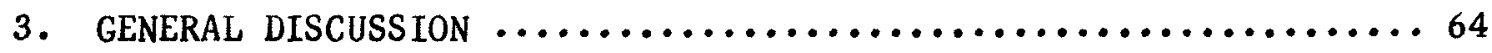

BIBLIOGRAPHY $\ldots \ldots \ldots \ldots \ldots \ldots \ldots \ldots \ldots \ldots \ldots \ldots \ldots \ldots \ldots, 68$ 


\section{INTRODUCTION}

The theory of regular semigroups is of paramount importance in the general framework of the structure of semigroups. Constructions of particular classes of regular semigroups (for example, completely simple and $\omega$-bisimple semigroups) have been made [2]. During the last few years the general construction of regular semigroups has gained the attention of clifford [4], Grillet [8], Namboodripad [11] etc. My own investigation in this direction resulted in a new property called the multiplication property enjoyed by regular semigroups. Surprisingly this property is not only enjoyed by regular semigroups, but also by many other important classes of semigroups; for instance, simple semigroups with identity, some important classes of semisimple semigroups (right regular, intra-regular and regular), and commutative principal ideal semigroups with identity. Hence one may ask how these different classes of semigroups can be differentiated in the general class of multiplication semigroups (semigroups satisfying the multiplication property); what is the particular place of regular semigroups in this general framework and how a general construction may be attempted? From the point of view of lattice theory, Anderson, one of Kaplansky's students wrote a thesis on multiplicative lattices [1].

It is well-known that regular semigroups are semisimple but semisimple semigroups are not necessarily regular. The property distinguishing the subclass of regular semigroups from the larger class of semisimple semigroups is not yet known. In the first Chapter of this dissertation we prove that the multiplication property distinguishes many important classes of semisimple semigroups from regular semigroups. Incidentally, 
we improve a classical result about the characterization of inverse semigroups which are unions of groups. We obtain the characterization of left cancellative right multiplication semigroups, not necessarily containing identity. This provides the construction of left cancellative right multiplication semigroups and shows that the only commutative cancellative multiplication semigroups containing identity are exactly of the form $\mathrm{N} \times \mathrm{G}$ where $\mathrm{N}$ is the additive semigroup of non-negative integers and $G$ is any arbitrary commutative group.

In the second chapter we show how the multiplication property distinguishes commutative principal ideal semigroups in the bigger class of commutative noetherian semigroups. We provide conditions under which a commutative noetherian semigroup is a regular semigroup, of course in terms of the multiplication property. We also detemine the structure of commutative multiplication semigroups in the noetherian case and the finite-dimensional case.

In the last chapter we discuss how the theory of commutative multiplication semigroups differs from the theory of multiplication rings developed by Gilmer [7] and Mott [10]. We also mention some unsolved problems for future study. 


\section{MULTIRLICATION SEMIGROUPS}

This chapter deals with non-commutative right multiplication semigroups. The concepts used in this chapter can be found in [2] and [3]. However, for completeness we mention definitions and properties of non-commutative semigroups which we need in the course of our discussion.

Throughout this chapter $S$ denotes an arbitrary semigroup.

\subsection{BASIC DEFINITIONS}

1.1 DEFINITION. An element $a \in S$ is an idempotent element if $a^{2}=a$;

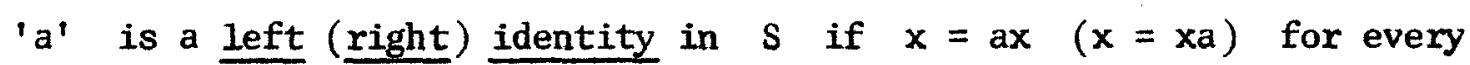
$x \in S$. ' $a$ ' is an identity in $S$ if it is both a left and right identity.

1.2 DEFINITION. An element $x \in S$ is a left cancellable element if $x a=x b$ for $a, b \in S$ implies that $a=b$; dually, if $a x=b x$ implies $a=b$, we say $x$ is a right cancellable element. A cancellable element is one which is both left and right cancellable. A semigroup all of whose elements are (left, right) cancellable is a (left, right) cancellative semigroup.

1.3 DEFINITION. An element a $\in S$ is (left, right, intra-) regular if $\left(a \in \mathrm{Sa}^{2}, \mathrm{a}^{2} \mathrm{~S}, \mathrm{Sa}^{2} \mathrm{~S}\right)$ a $\in$ aSa. $\mathrm{S}$ is a (left, right, intra-) regular semigroup if every element of $S$ is (left, right, intra-) regular.

1.4 DEFINITION. A subset $A$ of $S$ is a left (right) ideal of $S$ if for every $a \in A$ and $s \in S$ we have $s a \in A$ (as $\in A)$. A subset $A$ of $S$ is an ideal (also referred as a two-sided ideal) of $S$ if $A$ is both left and right ideal.

By a proper (left, right) ideal of $s$, we mean an (left, right) 
ideal which is properly contained in $S$.

1.5 NOTATION. For any (right, left) ideal $A$ of $S$ we denote $\bigcap_{n=1}^{\infty} A^{n}$ by $A^{\omega}$

1.6 DEFINITION. A proper (left, right) ideal A of $S$ is a maximal (left, right) ideal of $S$ if $A$ is not contained in any larger proper (left, right) ideal of $s$.

1.7 DEFINITION. An (left, right) ideal $A$ is an idempotent (left, right) ideal if $A=A^{2}$.

1.8 DEFINITION. An (left, right) ideal $A$ is finitely generated if $\left(A=\bigcup_{i=1}^{n}\left(x_{i} \cup S x_{i}\right), \quad A=\bigcup_{i=1}^{n}\left(x_{i} \cup x_{i} s\right)\right) A=\bigcup_{i=1}^{n}\left(x_{i} \cup x_{i} S \cup S x_{i} \cup S x_{i} s\right)$ where $x_{1}, \ldots, x_{n} \in S$ are a finite number of generators of $A$. In particular, if $(A=x \cup S x, A=x \cup x S) A=x \cup x S \cup S x \cup S x S$ we say $A$ is a principal (left, right) ideal.

For any $x \in S$, the principal (left, right) ideal generated by $x$ is denoted by $\left(s^{1} x, x s^{1}\right) s^{1} x s^{l}$.

1.9 DEFINITION. For any $x \in S$, the J-class containing $x$ is the set of all generators of $s^{1} \times s^{1}$.

1.10 DEEINITION. An ideal $A$ of $S$ is a prime ideal if asb $\subseteq A$ for $a, b \in S$ implies $a \in A$ or $b \in A$.

1.11 DEFINITION. $S$ is called (left, right) simple if it contains no proper (left, right) ideal. If $S$ contains a zero element 0 (i.e., $x \cdot 0=0 \cdot x=0$ for every $x \in S$ ), $S$ is called (left, right) 0-simple 
if (i) $s^{2} \neq 0$, and (ii) 0 is the only proper (left, right) ideal of $s$.

1.12 DEFINITION. $S$ is an inverse semigroup if (i) $S$ is regular, and (iì) any two idempotent elements of $\mathrm{S}$ commute with each other.

1.13 DEFINITION. $S$ is semisimple if $A=A^{2}$ for every ideal $A$ of $S$ (i.e., every ideal is idempotent). Trivially (left, right, intra-) regular semigroups are semisimple.

1.14 DEFINITION. $S$ is called a left (right) group if $S$ is left (right) simple and right (left) cancellative.

\subsection{GENERAL PROPERTIES}

1.15 PROPOSITION. Let $S$ be any semigroup. Then we have the following.

(i) Suppose $S$ contains maximal left ideals. Let $L^{*}$ be the intersection of all maximal left ideals. If a $\notin L^{*}$ and if $a \in$ as then $a \in$ aSa.

(ii) $a \in(a S)^{2}$ for every $a \in S$ if and only if every right ideal is idempotent. Further, if $S$ is simple, this is equivalent to the condition $a \in$ as for every $a \in S$.

(iii) Every right ideal of $S$ is idempotent if and only if $A=A B$ for any two right ideals $A$ and $B$ with $A \subseteq B$.

(iv) If $\mathrm{S}$ is regular then every right (left) ideal is idempotent.

(v) If $S$ is left cancellative with a unique maximal right ideal $M$ then (a) $M$ is two-sided, (b) further, if $M$ is finitely generated 
then $M \neq M^{2}$.

(vi) If $S$ is left cancellative with idempotents then (a) the set of idempotents is a subsemigroup of $s$, (b) $S$ is (right) simple or $\mathrm{S}$ contains a unique maximal (right) ideal containing every proper (right) ideal.

Proof. (i) Since a $\notin L^{*}$, there exists a maximal left ideal $\mathrm{N}$ not containing ' $a$ '. Therefore $s=\mathrm{N} \cup \mathrm{s}^{\mathrm{I}} \mathrm{a}$. By hypothesis, $\mathrm{a}=\mathrm{as}$ for some $s \in S$, and so $s \notin N$. Hence $s=N \cup s^{1} s=N \cup s^{1}$. Thus $s=a$ or $s \in S a$ which shows that $a \in a S a$.

(ii) Let $a \in(a S)^{2}$ for every $a \in S$. Let $A$ be a right ideal. For any $a \in A$, we have $a \in(a S)^{2} \subseteq A^{2}$ and thus $A=A^{2}$. Now suppose that every right ideal is idempotent. For any a $\in S$ we have a $U$ as $=(a \cup$ as $)(a \cup$ as $)$. We now have the following possibilities: $a=a^{2}$ or $a=a^{2} s$ for some $s \in S$ or $a=a$ ta for some $t \in S$. If $a=a^{2}$ then $a=a^{4} \in(a s)^{2}$. If $a:=a^{2} s$ then $a=\left(a^{2} s\right)(a s) \in(a s)^{2}$. Finally if $a=$ ata then $a=(a t)(a t a) \in(a s)^{2}$.

Further let $s$ be a simple semigroup with $a \in a S$. Then $a=a x$ for some $x \in S$. Clearly $x \in S=S a S$. Hence $a \in(a S)^{2}$. The other implication is evident.

(iii) Let $A$ and $B$ be any two right ideals of $S$ with $A \subseteq B$. Now $A=A^{2} \subseteq A B \subseteq A$ and so $A=A B$. The other implication is trivial. (iv) Let $A$ be a right (left) ideal of $S$. For any $a \in A$ we have $a=$ axa $\in A^{2}$, and thus $A=A^{2}$.

(v) Suppose $M$ is not two-sided. Then for some $s \notin M$ and $m \in M$ we have $s m \notin M$. Now $M U(\operatorname{sm} U \operatorname{sms})=s$, and so $s=s m$ or 
smt where $t \in S$. If $s=s m$ then $m$ is an idempotent which is a left identity and so $M=S$ which is not true. By a similar argument we arrive at a contradiction even in the other case. Hence $M$ is twosided.

Let $M$ befinitely generated. Suppose $M=M^{2}$. We then have $\bigcup_{i=1}^{n} x_{i} s^{1}=\left(\bigcup_{i=1}^{n} x_{i} s^{1}\right)\left(\bigcup_{i=1}^{n} x_{i} s^{1}\right)$ where $\left\{x_{i}\right\}_{1 \leq i \leq n}$ is theminimal set of generators and $x_{i} \in M$ for every $i$. Now $x_{1}=x_{1} s x_{j} t$ for some $s, t \in S$. Since $S$ is left cancellative it follows that $s x_{j} t$ is a left identity. In view of (i), sx $j \in M$ since $x_{j} \in M$. These facts show that $M=S$ which is not true. Hence $M \neq M^{2}$.

(vi) If $e$ is an idempotent then for every $a \in s$, ea $=e^{2} a$ and so $\mathrm{a}=\mathrm{ea}$ since $\mathrm{S}$ is left cancellative. In particular, for any two idempotents $e$ and $f$ we have $e=f e$ and $f=$ ef. Thus $E$, the set of all idempotents, is a subsemigroup of $\mathrm{S}$.

Suppose that $S$ is not (right) simple, Let $M=\underset{\alpha}{U} A_{\alpha}$ where each $A_{\alpha}$ is a proper (right) ideal of $S$. we show that $M$ contains no idempotents. Let $e$ be an idempotent in $M$. Then $e \in A_{\alpha}$ for some $\alpha$. Now since $S$ is left cancellative for every a $\in S$ we have $a=e a \in A_{\alpha}$ which shows that $A_{\alpha}=S$, which is not true. Hence $M$ contains no idempotents and so $M \neq S$. Now we show that $M$ is a maximal (right) ideal. Let $x \notin M$. Suppose that $s^{1} x s^{1}\left(x s^{1}\right) \neq s$. By choice of $M$ we have $s^{1} x s^{1}\left(x s^{1}\right) \subseteq M$, i.e., $x \in M$ which is a contradiction. Therefore, $s^{1} x s^{1}=S$. Finally we show that $M$ is the unique maximal (right) ideal. Let $\mathrm{T}$ be a maximal (right) ideal of $\mathrm{S}$ with $T \neq M$. Let $x \in T \backslash M$. As before $S^{1} x S^{1}\left(x S^{1}\right)=S \subseteq T$, which 
is not true. Thus $M$ is the unique maximal (right) ideal containing every proper (right) ideal of $\mathrm{S}$.

\subsection{PROPERTIES OF MULTIPLICATION SEMIGROUPS}

A semigroup $S$ is a right multiplication semigroup if for any pair of right ideals $A$ and $B$ with $A \subseteq B$, we have $A=C B$ for some right ideal $\mathrm{C}$ of $\mathrm{S}$. Dually, left multiplication semigroups can be defined.

\section{16 PROPOSITION. In a right multiplication semigroup $S$, the} following are tmue.

(i) $S=S^{2}, a \in$ aS for every $a \in S$ and $A=A S$ for every right ideal $A$ of $S$.

(ii) For any right ideal $A, A \subseteq S A$.

(iii) If $M$ is a maximal right ideal containing every proper right ideal then $M=M^{2}$ or $M$ is a two-sided ideal with $M=S M$. Further, if $M$ is unique with $M \neq M^{2}$ then $M=x S$ for some $x \in M \backslash M^{2}$.

(iv) If $S$ contains maximal left ideals and if $L^{*}=\emptyset$ where $L^{*}$ is the intersection of all maximal left ideals, then $S$ is regular. (v) If $S$ is semisimple then $M=M^{2}$ for every maximal right ideal $M$.

(vi) If $M$ is a unique maximal right ideal with every proper right ideal contained in $M$ and if $A$ is any proper right ideal then $A=M^{r}$ with $M^{r} \neq M^{r+1}$ or $A=A M$ with $A \subseteq M^{\omega}$.

(vii) If $\mathrm{S}$ is a semisimple semigroup such that every left ideal 
is two-sided, then $S$ is regular and left regular. Consequently, $S$ is a union of groups.

(viii) If $S$ contains a left cancellable element then $S$ contains an idempotent which is a left identity.

(ix) If $S$ is left - and right - cancellative then $S$ contains an identity and every right ideal is two-sided.

Proof. (i) Since $S \subseteq S$ we have $. S=$ AS for some right ideal $A$ and so $s=s^{2}$. For any $a \in S, a s^{1} \subseteq s$ so that for some right ideal $B$ we have $a S^{1}=B S=B S^{2}=\left(a S^{1}\right) S=a S$. Hence a $\in$ as for every a $\in S$. It is now immediate that $A=A S$ for every right ideal $A$.

(ii) For any ideal $A$, since $A \subseteq A$ we have $A=B A$ for some right ideal $B$ and thus $A \subseteq S A$.

(iii) Let $M$ be a maximal right ideal. Since $M \subseteq M$ we have $M=A M$ for some right ideal $A$. If $A \subseteq M$ then $M=M^{2}$. Suppose $A \nsubseteq M$. Then $A \cup M=S$. Now $A M U M^{2}=S M$, i.e., $M=S M$. clearly $M$ is a two-sided ideal. Next let $M$ be unique with $M \neq M^{2}$. For any $x \in M \backslash M^{2}$, since $x S \subseteq M$ we have $x S=A M$ for some right ideal $A$. Clearly $A=S$ since otherwise $x \in M^{2}$ which is not true. Thus $\mathrm{xS}=\mathrm{SM}=\mathrm{M}$.

(iv) Combining (i) of Proposition 1.15 and (i) the result follows.

(v) Suppose $M \neq M^{2}$ for some maximal right ideal $M$. Then from (iii) it follows that $M$ is a two-sided ideal. Since $S$ is semisimple we have $M=M^{2}$ which is a contradiction. Hence $M=M^{2}$.

(vi) Let $A$ be a proper right ideal of $S$. If $A \nsubseteq M^{(1)}$ then for some natural number $r, A \subseteq M^{r} \backslash M^{r+1}$. We then have $A=B M^{r}$ for 
some right ideal $B$ with $B \nsubseteq M$. Clearly $B=S$ and so $A=S M^{r}$. Also we have $M \neq M^{2}$. Now by (iii), $S M=M$ from which it follows that $A=M^{r}$ where $M^{r} \neq M^{r+1}$. Suppose $A \subseteq M^{W}$. Then $A=B_{1} M^{W}$ for some right ideal $B_{1}$. If $M^{r}=M^{r+1}$ then $A=B_{1} M^{r}=B_{1} M^{r+1}=A M$. Now suppose $M^{r} \neq M^{r+1}$ for any natural number $r$. Since $M^{\omega} \subseteq M$ we have $M^{\omega}=C M$ for some right ideal $C$. If $C=S$ then $M^{\prime \prime \prime}=S M=M$ by (iii) since $M \neq M^{2}$, which leads to a contradiction. Now $C \subseteq M$. If $C \subseteq M^{r} \backslash M^{r+1}$ then as before $C=M^{r}$ so that $M^{(\omega)}=M^{r+1}$ which is again a contradiction. Thus $C \subseteq M^{\omega}$. Hence $M^{\omega} \subseteq M^{\omega \prime} M \subseteq M^{\omega}$ and so $M^{(4)}=M^{(\prime)} M$. We thus have $A=B_{1} M^{(\omega)}=B_{1} M^{(\prime)} M=A M$, where $A \subseteq M^{\omega}$.

(vii) For any $a \in S$ we have $a S \subseteq$ SaS by (ii). Now aS $=B(S a S)$ for some right ideal $B$. Since $S$ is semisimple it now follows that $\mathrm{aS}=\mathrm{B}(\mathrm{SaS})=\mathrm{B}(\mathrm{SaS})(\mathrm{SaS})=\mathrm{aS}(\mathrm{SaS}) \subseteq \mathrm{aSaS}$. Since every left ideal is

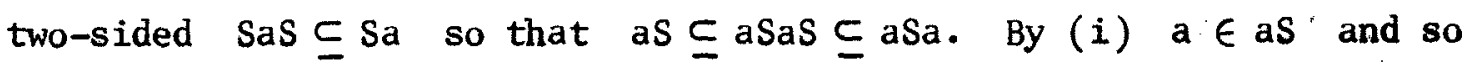
$a \in$ aSa. Thus $S$ is a regular semigroup. Further, since the left ideal a $U S a$ is two-sided we have as $\subseteq$ a U Sa. Thus $a \in a S a \subseteq(a \cup S a) a=a^{2} \cup S^{2}$. clearly $s$ is a left regular semigroup. It then follows from Theorem 4.3 of [2] that $S$ is a union of groups.

(viii) Let $x \in S$ be a left cancellable element. By (i), $x=x s$ for some $s \in S$ so that $x s=x s^{2}$ and thus $s=s^{2}$ since $x$ is left cancellable. We note that $s$ is a left cancellable element. For, if $s a=s b$ for some $a, b \in S$ then $x s a=x s b$, i.e., $x a=x b$ and so $a=b$. Now for any $y \in S$ we have sy $=s^{2} y$. Then $y=$ sy since $s$ is left cancellable. Thus the idempotent element $s$ is a left identity in $\mathrm{s}$. 
(ix) Since $S$ is left cancellative, $S$ contains a left identity by (viii). If $s \in S$ is a left identity element in $s$ then $x s=x s^{2}$ for every $x \in S$. Since $S$ is right cancellative $x=x s$ which shows that $s$ is also a right identity in $s$. Now let $A$ be any right ideal in $S$. For any a $\in A$, a $U$ aS $=c(a U$ aS $)$ for some right ideal c. Now $a=x a$ or $x a t$ for some $x \in C$ and $t \in S$. If $a=x a$ then sa $=a=x a$ and so $s=x$ since $s$ is right cancellative. Now $s=x \in C$ and thus $c=s$. We now have aa $U$ as $=S(a U$ as $)$ which shows that $S A \subseteq A$, i.e., $A$ is a two-sided ideal in this case. Now suppose $a=x a t$. Since $x \in C, x S \subseteq C$ and so $x S a \subseteq a U$ aS. For any $u \in S$ we have $x u a=a$ or $a u^{\prime}, \dot{u}^{\prime} \in S$. In the first case $x u a=a=x a t$ and so $u a=a t \in A$ since $S$ is left cancellative. In the second case, $x u a=a u^{\prime}=x a t u^{\prime}$ and so $u a=a t u^{\prime} \in A$. In either case Sa $\subseteq A$, which shows that $S A \subseteq A$. Thus $A$ is a two-sided ideal.

1.17 REMARK. If $\mathrm{S}$ is a left cancellative right multiplication semigroup then by (viii) in the agove, $S$ should contain idempotents. Hence the semigroups of Baer-Levi type, which are left simple and left cancellative, are not right multiplication semigroups.

\subsection{SEMISIMPLE SEMIGROUPS}

In this section we show that simple semigroups containing identity, regular semigroups and right regular semigroups are right multiplication semigroups. But left regular semigroups and left cancellative, left simple (Baer-Levi type) semigroups are not right multiplication semigroups. This shows that not all semisimple semigroups are right multiplication 
semigroups. We obtain necessary and sufficient conditions for semisimple semigroups to be right multiplication semigroups and we obtain the structure of left regular and intra-regular semigroups which are right multiplication semigroups. There exist semisimple semigroups which are right multiplication semigroups without being regular. In this connection we provide some sufficient conditions for regularity.

1.18 THEOREM. Let $S$ be a simple semigroup. Then $S$ is a right multiplication semigroup if and only if a $\in$ as for every a $\in S$.

Proof. Let $S$ be a simple semigroup with a $\in$ aS for every a $\epsilon$. Let $A$ and $B$ be any two right ideals with $A \subseteq B$. Now $A S \subseteq A$ and so $A S B \subseteq A B$. Since $S$ is simple we have $S B=S$. We thus have $A \subseteq A S \subseteq A B \subseteq A$. Hence $A=A B$ which shows that $S$ is a right multiplication semigroup. The other implication is evident by ( $i$ ) of Proposition 1.16 .

1.19 PROPOSITION. In a semigroup $S$, if $a \in(a S)^{2}$ for every a $\in S$ then $S$ is a right multiplication semigroup.

Proof. Let $A$ and $B$ be any two right ideals of $S$ with $A \subseteq B$. For every $a \in A$, we have $a \in(a S)^{2} \subseteq A B$. Thus $A \subseteq A B \subseteq A$, i.e., $A=A B$ which shows that $S$ is a right multiplication semigroup.

1.20 COROLLARY. If $\mathrm{S}$ is a right regular semigroup or a regular semigroup then $S$ is a right multiplication semigroup.

Proof. For every $a \in S$ we have $a=a^{2} t$ or $a=a t a$ for some $t \in S$. Clearly $a \in(a S)^{2}$ in either case. Now the corollary follows 
from the above Proposition.

1.21 THEOREM. The following are equivalent for a semigroup $\mathrm{s}$.

(i) $\mathrm{S}$ is a left simple right multiplication semigroup.

(ii) $\mathrm{S}$ is a left simple semigroup containing idempotents.

(iii) $\mathrm{S}$ is a left group.

Proof. (i) $\Rightarrow$ (ii). For any $a \in S$ we have $a=a x$ for some $x \in S$ by (i) of Proposition 1.16. Since $S$ is left simple we have $x \in S=S a$. Therefore $a \in$ aSa. Hence $S$ contains idempotents.

(ii) $\Rightarrow$ (iii) follows from Theorem 1.27 of [2].

(iii) $\Rightarrow(i)$. Since left groups are regular, the result follows from Corollary 1.20 .

1.22 THEOREM. A semisimple semigroup $S$ is a right multiplication semigroup if and only if $a \in(a S)^{2}$ for every $a \in S$.

Proof. Let $S$ be a right multiplication semigroup. In view of (ii) of Proposition 1.16, we may write aS $\subseteq$ SaS for any a $\in S$. Hence $\mathrm{aS}=\mathrm{T}(\mathrm{SaS})$ for some right ideal $\mathrm{T}$. Since every ideal is idempotent in a semisimple semigroup, we have aS $=\mathrm{T}(\mathrm{SaS})=\mathrm{T}(\mathrm{SaS})(\mathrm{SaS})=\mathrm{aS}(\mathrm{SaS}) \subseteq$ $\subseteq$ aSaS. By (i) of Proposition 1.16, a $\in$ as and so $a \in(a S)^{2}$. The other implication follows from Proposition 1.19.

1.23 THEOREM. Let every right ideal be a two-sided ideal in a semigroup $S$. Then $S$ is a semisimple right multiplication semigroup if and only if $S$ is right regular.

Proof. Let $S$ be a semisimple right multiplication semigroup. 
Then for every $a \in S$ we have $a \in(a S)^{2}$ by Theorem 1.22. Since every right ideal is two-sided it follows that a $\in$ aSas $\subseteq a^{2} \mathrm{~s}$. Conversely, suppose $\mathrm{S}$ is right regular. Let $\mathrm{A}$ be any ideal of $\mathrm{S}$. For any $a \in A, a \in a^{2} S=a \cdot a S \subseteq A^{2} \subseteq A$, i.e. $A=A^{2}$. Thus $S$ is semisimple. Also $S$ is a right multiplication semigroup by Corollary 1.20 .

1. 24 REMARK. If we drop the assumption that every right ideal is two-sided, the theorem need not be true. For, there exist regular semigroups (hence semisimple right multiplication semigroups) which are not the union of groups. Clearly these are not right regular since otherwise by Theorem 4.3 of [2], they should be a union of groups.

1.25 THEOREM. If every left ideal is two-sided in a semigroup $S$ then $S$ is a semisimple right multiplication semigroup if and only if $S$ is a union of groups.

Proof. Let $S$ be a semisimple right multiplication semigroup. Now for any $a \in S$ we have $a \in(a S)^{2}$ by Theorem 1.22. Clearly a $\in$ aSaS $\subseteq$ aSa since every left ideal is two-sided. Also as $\subseteq$ Sa and so $a \in \mathrm{Sa}^{2}$. Thus $s$ is regular and left regular. It follows in view of Theorem 4.3 of [2] that $S$ is a union of groups. Converse follows from Theorem 4.3 of [2] and Corollary 1.20 and the fact that regular semigroups are semisimple.

One of the classical results mentioned in Example 2 of [2, page 129] is that a semigroup is a semilattice of groups if and only if it is an inverse semigroup with every one-sided ideal as two-sided. Now we improve this result. 
1.26 THEOREM. Let $S$ be a semigroup in which every one-sided ideal is two-sided. Then $\mathrm{S}$ is a semisimple right multiplication semigroup if and only if $S$ is a semilattice of groups.

Proof. By combining the result in Example 2 [2, page 129] and Theorem 1.25, the proof follows.

1.27 REMARK. We note that semisimple right multiplication semigroups are not necessarily regular. For example the semigroup $\mathrm{C}\left(\mathrm{s}^{1}\right)$, generated by $s^{1} \cup\{a, b\}$ where $s^{1}$ is any semigroup, $a, b \notin s^{1}$ subject to the relations $a b=1, a s=a, s b=b$ for any $s \in s^{1}$, is not a regular semigroup if $\mathrm{s}^{1}$ is not regular, in view of Theorem 8.48 of [3]. But this is a simple semigroup containing identity [3, page 109] and hence by Theorem 1.18, this is a right ( and left) multiplication semigroup. In view of this, a stronger hypothesis is needed even for semisimple two-sided multiplication semigroups to be regular. In the following, a sufficient condition is given.

1.28 THEOREM. If $S$ is a semisimple right multiplication semigroup such that $a b \in S b a$ for every $a, b \in S$, then $S$ is a regular semigroup.

Proof. By Theorem 1.22 for any $a \in S$ we have $a=$ asat for some $s$ and $t \in S$. 1 Now at $\in$ Sta and so at $=x$ ta for some $x \in S$. Clearly $a \in$ aSa and hence $S$ is regular.

1.29 THEOREM. Let $S$ be a left regular or intra-regular semigroup. Then $S$ is a right multiplication semigroup if and only if $S$ is a 
union of simple semigroups $S_{\alpha}$ with $x \in x S_{\alpha}$ for every $x \in S_{\alpha}$.

Proof. Let $S$ be a left regular, right multiplication semigroup. We show that $S$ is intra-regular. By (i) of Proposition 1.16, for any $a \in S$ we have $a \in$ as. But left regularity of $S$ shows that $a \in \mathrm{Sa}^{2}$ from which it follows that $a \in \mathrm{Sa}^{2} \mathrm{~S}$. Thus $\mathrm{S}$ is intraregular. Now by Theorem 4.4 of [2], $\mathrm{S}$ is a union of simple semigroups $S_{\alpha}$, where each $S_{\alpha}$ is a J-class. Next we note that $S$ is semisimple. For, let $A$ be any ideal. Since $S$ is intra-regular, for any $x \in A$ we have $x \in S x^{2} S=(S x)(x S) \subseteq A^{2}$, i.e., $A=A^{2}$. Hence $S$ is semisimple. Now by Theorem 1.22 for every $a \in S_{\alpha}$ we have $a=$ axay for some $x$ and $y \in s$. Clearly $s^{1} s^{1}=s^{1}$ xays $s^{1}$. Thus xay $\in s_{\alpha}$ and hence $a \in a S_{\alpha}$.

Conversely, for every $a \in S$ we have $a \in S_{\alpha}$ for some $\alpha$ and $a \in a s_{\alpha}=a\left(s_{\alpha} a s_{\alpha}\right)$ since $s_{\alpha}$ is a simple semigroup. Now by Proposition $1.19, \mathrm{~S}$ is a right multiplication semigroup.

\subsection{LEFT CANCELLATIVE SEMIGROUPS}

In this section we obtain conditions for a left cancellative semigroup (not necessarily containing identity) to be a right multiplication semigroup. This generalizes our result published in [5] in the commutative case.

1.30 THEOREM. Let $S$ be a semigroup with a unique maximal right ideal $M$ such that $A \neq A M$ for every proper right ideal $A$. Suppose that every proper right ideal is contained in $M$. Then $S$ is a 
right multiploication semigroup if and only if $S$ satisfies the following conditions.

(i) Every proper right ideal is of the form $M^{r}$;

(ii) $\mathrm{s}=\mathrm{s}^{2}$;

(iii) $M=S M=M S$.

Furthermore, $M=x S^{1}$ for some $x \in S$ and every element a $\in M$ is of the form $x^{r} u$ for some natural number $r$, where $u \notin M$.

Proof. Let $S$ be a right multiplication semigroup. By hypothesis $M \neq M^{2}$ and so $M=S M$ by (iii) of Proposition 1.16. We claim that $M^{\omega}=\emptyset$. Suppose $M^{\omega} \neq \emptyset$. Since $M^{\omega} \subseteq M$ we have $M^{\omega}=C M$ for some right ideal $C$. In view of (vi) of Proposition 1.16 we have $c=M^{x}$ for some natural number $r$. But then $M^{\omega}=M^{r+1}$, i.e., $M^{r+1}=M^{r+2}$ which is not true. Hence $M^{\omega}=\emptyset$. Now by (vi) of Proposition 1.16 every proper right ideal is of the form $M^{r}$ for some natural number $r$. (ii) and (iii) follow from (i) and (iii) of Proposition 1.16. A direct verification yields the converse.

Finally, since $M \neq M^{2}$ it follows from (iii) of Proposition 1.16 that $M=x S$ where $x \in M \backslash M^{2}$. Now, if $a \in M$ then $a=x s_{1}$ for some $s_{1} \in s$. If $s_{1} \notin M$ we are done. If $s_{1} \in M$ then $s_{1}=x s_{2}$ for some $s_{2} \in \mathrm{s}$. Proceeding, if every $s_{i} \in M$ then $a=x s_{1}=x^{2} s_{2}=\ldots$, and so $a \in M^{\omega}=\varnothing$. Hence $a=x^{r}$ s for some natural number $r$, where $\mathbf{s} \notin \mathrm{M}$.

1.31 DEFINITION. A semigroup $S$ is called $Q^{*}$-simple if it contains no proper prime ideals.

We note that $Q^{*}$-simple semigroups are archimedian semigroups as 
defined in $[12$, p. : 49].

We now discuss the structure of left cancellative right multiplication semigroups.

1.32 THEOREM. Let $S$ be a left cancellative semigroup. Then $S$ is a right multiplication semigroup if and only if $S$ has idempotents and $S$ is one of the following types.

(i) S is a right simple semigroup.

(ii) $S$ has a unique maximal right ideal $M$ which is also twosided with $M=M S$, and such that every proper right ideal is of the form $M^{r}$ and thus is two-sided.

In the second case $M=x s^{1}$ for some $x \in M \backslash M^{2}$ and every element in $S$ is of the form $x^{r} u$, where $u \notin M$ and $r$ is a non-negative integer. Furthermore, $S$ is an extension of a $Q^{*}-$ simple semigroup by a right o-simple semigroup.

Proof. If $S$ is a right multiplication semigroup then by (viii) of Proposition 1.16, $S$ contains idempotents. If $S$ is not right simple, by (vi) of Proposition 1.15, $\mathrm{S}$ contains a unique maximal right ideal $M$ such that every proper right ideal is contained in $M$. By ( $v$ ) of Proposition 1.15, $M$ is two-sided. Now we show that $A \neq A M$ for every proper right ideal A. Suppose $A=A M$ for some proper right ideal $A$. Then for $a \in A, a S^{1}=T A$ for some right ideal $T$. Thus $\mathrm{aS}^{1}=\mathrm{TA}=\mathrm{TAM}=\left(\mathrm{aS}^{1}\right) \mathrm{M} \subseteq \mathrm{aM}$ which shows that $\mathrm{a}=$ am for some $\mathrm{m} \in M$. Clearly im is an idempotent and thus a left identity, since $S$ is left cancellative. Hence $M=S$ which is not true. Therefore, $A \neq A M$ for every proper right ideal A. Since $S$ has idempotents which are 
obviously left identities, it follows that $S=s^{2}$ and $M=S M$. Now from the proof of Theorem 1.30, every statement of the theorem except the last is evident.

Finally, since $M$ is a two-sided ideal which is also the unique maximal right ideal, $S / M$ is clearly a right o-simple semigroup. Now we claim that the subsemigroup $M$ has no proper prime ideals. Let $P$ be a prime ideal of $M$ with $P \neq M$. Since $P M$ is a proper (right) ideal of $S$, we have $P M=M^{r} \Subset P$ and hence $P=M$ which is a contradiction. Thus $\mathrm{S}$ is an extension of a $Q^{*}-$ simple semigroup by a right o-simple semigroup.

1.33 COROLLARY. Let $S$ be a left and right cancellative semigroup which is not a group. Then $S$ is a right multiplication semigroup if and only if (i) S contains identity, (ii) $S$ has a unique maximal right ideal of the form $x S$ for some $x \in S$ which is two-sided and every proper right ideal is of the form $x^{r}$ for some natural number $r$, and thus is two-sided.

Proof. By (ix) of Proposition 1.16, $S$ contains identity. If $S$ is right simple then $S$ is a group which is not true. Now the Corollary follows from the proof of the above theorem.

As an immediate consequence of Theorem 1.32 we have the following in the commutative case [5].

1.34 COROLLARY. A commutative semigroup $S$ (which is not a group) is a cancellative multiplication semigroup if and only if $\mathrm{S}$ is isomorphic to $N \times G$ where $N$ is the additive semigroup of non-negative integers and $G$ is any group. 
1.35 THEOREM. For a left cancellative semigroup $S$, the following are equivalent.

(i) $\mathrm{S}$ is a semisimple right multiplication semigroup.

(ii) $S$ is a simple semigroup with a $\in$ aS for every a $\in S$. .

Proof. (i) $\Rightarrow(i i)$. Let $A$ be a proper ideal of $s$. If a $\in A$ then $a=$ asat for some $s, t \in S$ by Theorem 1.22. Since $s$ is left cancellative it follows that sat is an idempotent which is also a left identity in $S$. But sat $\in A$ which shows that $A=S$ which is not true. Hence $S$ is simple. We have $a \in a S$ for every a $\in S$ by (i) of Proposition 1.16.

(ii) $\Rightarrow$ (i) follows from Theorem 1.18 .

1.36 REMARK. The following example shows that left cancellative condition is essential in the above theorem. Let $c\left(s^{1}\right)$ be the semigroup considered in Remark 1.27 which is a simple semigroup with identity. Let $A$ and $B$ be two copies of $C\left(S^{1}\right)$ and $T=A \cup B \cup 0$ where $a b=b a=0$ for every $a \in A$ and $b \in B$ and retain multiplications in $A$ and $B$. The only ideals of $T$ are $(0), A, B, A \cup B$ and $T$. $\mathrm{T}$ is a semisimple right multiplication semigroup. But $\mathrm{T}$ is not simple. 


\section{COMMUTATIVE MULTIPLICATION SEMIGROUPS}

In this chapter we discuss the structure of multiplication semigroups in the commutative case. For this we need some idealtheoretic eoncepts and properties of commutative semigroups. Most of these concepts are analogous to those in commutative ring theory. One can find some of these properties in [2] and [14]. However, for quick reference we mention all the properties needed for our discussion.

Throughout $S$ denotes a commutative semigroup which is not a group and hence $S$ contains proper ideals.

\subsection{BASIC DEFINITIONS AND NOTATION}

We reeall the definition of a prime ideal (1.10) which is equivalent to the following in the commutative case.

2.1 DEFINITION. An ideal $\mathrm{P}$ of $\mathrm{S}$ is a prime ideal, if whenever $\mathrm{A}$ and $\mathrm{B}$ are any two ideals with $\mathrm{AB} \subseteq \mathrm{P}$ and $\mathrm{A} \nsubseteq \mathrm{P}$ then $\mathrm{B} \subseteq \mathrm{P}$. Equivalently, $\mathrm{P}$ is a prime ideal, if whenever $\mathrm{a}, \mathrm{b} \in \mathrm{S}$ with $a b \in P$ and $a \notin P$ then $b \in P$.

2.2 DEFINITION. Let $A$ be an ideal contained in a prime ideal $P$. We say that $P$ is a minimal prime divisor of $A$ if whenever $A \subseteq Q \subseteq P$ for some prime ideal $Q$ then $Q=P$.

2.3 DEFINITION. For any ideal $A$ of $S$, the radical of $A$, denoted by $\sqrt{\mathrm{A}}$, is the intersection of all its minimal prime divisors. Equivalently, $\sqrt{\mathrm{A}}=\left\{\mathrm{x} \in \mathrm{S} \mid \mathrm{x}^{\mathrm{n}} \in \mathrm{A}\right.$ for some natural number $\left.\mathrm{n}\right\}$; 
also $\sqrt{A}$ is the intersection of all prime ideals containing $A$.

2.4 DEFINITION. An ideal $Q$ of $S$ is a prinary ideal, if whenever $A$ and $B$ are any two ideals with $A B \subseteq Q$ and $A \nsubseteq Q$ then $B \subseteq \sqrt{Q}$. Equivalently, $Q$ is a primary ideal if whenever $a, b \in S$ with $a b \in Q$ and $a \in Q$ then $b \in \sqrt{Q}$.

We note that the radical of a primary ideal is a prime ideal. We say $Q$ is P-primary for some prime ideal $P$, if $Q$ is primary with $\sqrt{Q}=\mathrm{P}$.

By a proper ideal we mean an ideal different from $S$. The symbol $<$ will mean 'properly contained in'.

2.5 DEFINITION. Consider a chain $\mathrm{P}_{0}<\mathrm{P}_{1}<\ldots<\mathrm{P}_{\mathrm{r}}$ of $\mathrm{r}+1$ proper prime ideals of $S$. The length of such a chain is the integer $r$. The dimension of $S$, denoted by $\operatorname{dim} S$, is the supremum of the lengths of all chains of distinct proper prime ideals of $S$. If $S$ contains no proper prime ideals then $\operatorname{dim} S$ is undefined.

We note that $\operatorname{dim} S=\infty$ or $\mathbf{n}$ where $\mathbf{n}$ is a non-negative integer. In the former case, for every non-negative integer $n$, we can find a chain of distinct proper prime ideals of length greater than $n$. In the latter case, there exists a chain of distinct proper prime ideals of length $n$ and no such chain of length greater than $n$ exists.

2.6 DEFINITION. If $\mathrm{P}$ is a minimal prime divisor of an ideal $\mathrm{A}$ of $S$, then the intersection of all P-primary ideals containing $A$ is called the isolated P-primary component of A.

2.7 DEFINITION. For any ideal $A$ of $S$, we define Ker $A$ as the 
intersection of all the isolated P-primary components of $A$, as $P$ runs through the minimal prime divisors of $A$.

To any semigroup $S$ without an identity, we can adjoin an identity by taking the set $S \cup 1$, where 1 is some element not contained in S. We define multiplication in $s$ by: $s l=1 s=s$ for all $s \in S \cup l$, while retaining the multiplication among the elements of $S$.

2.8 NOTATION. Let $S$ be a semigroup. If $S$ has an identity, set $s^{1}=S$. If $s$ does not have an identity, let $s^{1}$ be the semigroup $s$ with an identity adjoined, usually denoted by the symbol 1 .

2.9 DEFINITION. A semigroup containing identity is called a monoid. Evidently $\mathrm{s}^{1}$ is a monoid.

2.10 DEFINITION. Let $\mathrm{S}$ be a monoid and $\mathrm{P}$ be a prime ideal of $\mathrm{S}$. Consider the set of all ordered pairs $(a, b)$ with $a \in S$ and $b$ is a cancellable element in $S \backslash P$. Define an equivalence relation ' $~$ by: $(a, b) \sim(c, d)$ if and only if $a d=b c$. Denote by $a / b$. the equivalence class containing $(a, b)$. Now we define the semigroup of fractions $:_{\mathrm{S}}=\{\mathrm{a} / \mathrm{b}: \mathrm{a} \in \mathrm{S}, \mathrm{b} \in \mathrm{S} \backslash \mathrm{P}$ is a cancellable element $\}$. By defining a map $\eta: s \rightarrow s_{p}$ by $\eta(s)=s s / 1: \quad(s \in s), \quad s$ can be identified with a subsemigroup of $S_{p}$. For any ideal $A$ of $S$, the ideal generated by $\eta(A)$ in $S_{p}$ is denoted by $A_{p}$. Also $A S_{p} \cap S$ is an ideal of $S$. Moreover, if $A \subseteq P$ then $A S \cap \cap$ is an ideal of $S$ with radical $P$.

2.11 DEFINITION. For any monoid $S$, we define its total quotient monoid $T=r\{a / b: a, b \in S$ and $b$ is a cancellable elementiuin $S\}$. 
2.12 NOTATION. If $A$ and $B$ are any two ideals of $S$, we write $A: B=\{x \in S \mid x B \subseteq A\}$. We note that the set $A: B$ defined above is actually an ideal of $S$. We recall from first chapter the definition of a finitely generated (principal) ideal of $S$.

2.13 DEFINITION. $S$ is a noetherian (principal ideal) semigroup, if every ideal of $S$ is finitely generated (principal). We note that noetherian condition is equivalent to the ascending chain condition on ideals of $S$.

For completeness, we shall prove some facts (proofs being similar to those in commutative ring theory) which are essential for our discussion in the later part of this chapter.

\subsection{GENERAL PROPERTIES OF COMMUTATIVE SEMIGROUPS}

2.14 THEOREM. The following are true in a commutative semigroup $S$. (i) $\mathrm{s}^{1}$ has a unique maximal ideal containing every proper ideal of $S$.

(ii) If $P$ is a prime ideal of $S$ containing an ideal $A$, then $\mathrm{P}$ contains a minimal prime divisor of A. Consequently, every ideal of $S$ has a minimal prime divisor.

(iii) $S$ is regular if and only if every ideal is idempotent. (iv) If $S$ is a principal ideal semigroup then the set of ideals of $S$ is linearly ordered.

(v) Suppose that $S$ does not contain an identity. Then there is a one-to-one correspondence between (prime, primary) ideals of $S$ 
and proper (prime, primary) ideals of $\mathrm{s}^{1}$; moreover, $s$ is noetherian if and only if $\mathrm{s}^{1}$ is.

(vi) If $S$ is a noetherian semigroup then every ideal of $S$ is a finite intersection of primary ideals.

(vii) If $S$ is a noetherian semigroup then every ideal of $S$ has only a finite number of distinct minimal prime divisors.

(viii) If $A$ is a finitely generated idempotent ideal, then $A=\bigcup_{i=1}^{n} e_{i} s$ where $e_{i}^{\prime} s$ are idempotent elements in $A$.

(ix) If $\left\{\mathrm{P}_{\alpha}\right\}$ is the set of idempotent ideals of $\mathrm{S}$ each of which is not principal, then $U_{\alpha} P_{\alpha}$ is an idempotent ideal of $S$ which is not principal.

(x) Suppose that $S$ satisfies the following two conditions. (a) Every ideal has only a finite number of distinct minimal prime divisors and is a product of its minimal prime divisors. (b) For any prime ideal $P, P^{n}$ is P-primary for every natural number $n$. Then for any pair $(P, A)$ where $P$ is asprime ideal properly contained in the ideal $A$, we have $P=P A$.

(xi) Suppose that every ideal of $S$ with prime radical is primary and that every primary ideal is some power of its radical. Let $P$ be a prime ideal with powers of $P$ properly descending. Then $P^{\omega}$ is empty or a prime ideal with $\mathrm{P}^{(1)}<\mathrm{P}$.

(xii) Suppose that every ideal of $S$ with prime radical is primary and that every primary ideal is some power of its radical. If $A$ is an ideal of $S$ with a minimal prime divisor $P$, then the isolated P-primary component of $\mathrm{A}$ is some power of $\mathrm{P}$. 
(xiii) If $A=\operatorname{Ker} A$ for every ideal $A$ of $S$, then every ideal with prime radical is primary.

(xiv) If $S=s^{2}$ then for any maximal ideal $M$, we have $M=M^{2}$ or $M=M^{2} U M^{*}$ where $M^{*}$ is the intersection of all maximal ideals of $S$.

Proof. (i) Since $S$ is not a group there exist proper ideals in $\mathrm{s}^{l}$. If $\left\{A_{\alpha}\right\}$ is the set of all proper ideals of $\mathrm{s}^{l}$ then $\cup_{\alpha} A_{\alpha}$ is the unique maximal ideal of $s^{1}$, which is evidently a proper ideal of $\mathrm{s}^{1}$ containing every proper ideal of $\mathrm{s}$.

(ii) Let 6 be the set of all prime ideals contained in $P$, each of which contains A. Since $P \in 6, \zeta \neq \emptyset$. Let $\supseteq$ (reverse of set-inclusion) be the partial ordering in 5. Let $\left\{A_{\alpha}\right\}$ be a chain in 6. Then $\bigcap_{\alpha} A_{\alpha} \mid$ is a prime ideal. For, let $a b \in \bigcap_{\alpha} A_{\alpha}$ and suppose a $\notin \cap_{\alpha} A_{\alpha^{*}}$ Then a $\notin A_{\alpha}$ for some $\alpha$ and so $b \in A_{\alpha}$. Consider $A_{\beta} \neq A_{\alpha}$ in the chain $\left\{A_{\alpha}\right\}$. Now $A_{B} \subseteq A_{\alpha}$ or $A_{\alpha} \subseteq A_{B}$. If $A_{\beta} \subseteq A_{\alpha}$, then $a \notin A_{\beta}$ and so $b \in A_{\beta}$. If $A_{\alpha} \subseteq A_{\beta}$, then $b \in A_{\beta}$. Thus $b \in \underset{\alpha}{\cap} A_{a}$ and hence $\bigcap_{\alpha} A_{\alpha}$ is a prime ideal. Clearly $\bigcap_{\alpha} A_{\alpha}$ is a maximal element for the chain $\left\{A_{\alpha}\right\}$. Now by Zorn's lemma, there exists a maximal element of $\zeta$ which is a minimal prime divisor of $A$ contained in $P$. Thus $P$ contains a minimal prime divisor of $A$. Now, $S$ considered as a prime ideal, contains every ideal and thus contains a minimal prime divisor of every ideal. Thus every ideal has a minimal prime divisor.

(iii) Let $S$ be a regular semigroup. Suppose there is an ideal $A$ with $A \neq A^{2}$. Let $a \in A \backslash A^{2}$. Now $a=a^{2} s$ for some $s \in S$. clearly a $\in A^{2}$, which is a contradiction. Therefore, every ideal is 
idempotent in a regular semigroup. Conversely, for any element $a \in S$ we have $a s^{1}=a^{2} s^{1}$. Now $a=a^{2} s$ for some $s \in S$ and hence $s$ is $a$ regular semigroup.

(iv) Let $a S^{1}$ and $b^{1}$ be any two ideals of $S$. Now $\mathrm{as}^{1} \mathrm{UbS} \mathrm{S}^{1}=\mathrm{cS}^{1}$ for some $\mathrm{c} \in \mathrm{s}$, since $\mathrm{S}$ is a principal ideal semigroup. Then $c \in a S^{1}$ or $b S^{1}$ which shows that $c s^{1} \subseteq a s^{1}$ or $b s^{1}$, i.e., $\mathrm{bs}^{1} \subseteq \mathrm{as}^{1}$ or $a s^{1} \subseteq \mathrm{bs}^{1}$. Thus the set of ideals of a principal ideal semigroup is linearly ordered.

(v) Trivial.

(vi) We need the definition of an irreducible ideal, here. An ideal $A$ is called an irreducible ideal if $A$ cannot be written as a finite intersection of ideals that contain A properly. Now we prove the pxoperty mentioned above for noetherian semigroups. We first show that every ideal is a finite intersection of irreducible ideals. Suppose there is an ideal which is not a finite intersection of irreducible ideals. Consider the set of all ideals which cannot be written as a finite intersection of irreducible ideals. Since $S$ is noetherian, there exists a maximal element in this set, say L. Clearly $L$ is itself not irreducible. So we can write $L=C \cap D$, where $C$ and $D$ are ideals that contain L properly. By maximality of L, clearly C and $\mathrm{D}$ can be expressed as finite intersections of irreducible ideals. Thus $L$ is a finite intersection of irreducible ideals, which is a contradiction. Hence every ideal is a finite intersection of irreducible ideals. Now we show that every irreducible ideal is a primary ideal, which completes the proof. Let $A$ be an irreducible ideal. Suppose $A$ is not primary. Then for some $x \notin A$ and $y \notin \sqrt{A}$ we have $x y \in A$. 
Consider the chain $A: y S^{1} \subseteq A: y^{2} S^{1} \subseteq \ldots$. Since $S$ is noetherian, this chain terminates, i.e., for some $n$ we have $A: y^{n} S^{1}=A: y^{n+1} S^{I}=A: y^{n+2} S^{1}$ etc. Now we claim that $A=\left(A \cup x S^{1}\right) \cap\left(A \cup y^{n^{1}} S^{1}\right):$ Evidently $A \subseteq\left(A \cup x S^{1}\right) \cap\left(A \cup y^{n^{1}}\right)$. Now let $a \in\left(A \cup x S^{1}\right) \cap\left(A \cup y^{n} S^{1}\right)$. We have the following possibilities: $a \in A$ or $a=x=y^{n}$ or $a=x=y^{n} t \quad$ or $a=x s=y^{n}$ or $a=x s_{1}=y^{n} t_{1}$ for some $t, s, s_{1}, t_{1} \in S$. If $a \in A$, we are done. If $a=x=y^{n}$ then $a y=x y=y^{n+1} \in A$, since $x y \in A$. But this is not possible since $y \notin \sqrt{A}$. Hence this case does not arise. If $a=x=y^{n} t$ then ay $=x y=y^{n+1} t \in A$ since $x y \in A$. Thus $t \in \cdot A: y^{n+1} s^{1}=A: y^{n} s^{1}$ which shows that $\operatorname{ty}^{n} \in A$. Therefore, $a \in A$ in this case. If $a=x s=y^{n}$ then ay $=x y s=y^{n+1} \in A$ since $x y \in A$. But $y \notin \sqrt{A}$ so this is impossible. Finally if $a=x s_{1}=y^{n} t_{1}$ then $a y=x y s_{1}=$ $=y^{n+1} t_{1} \in A$ since $x y \in A$. Now $t_{1} \in A: y^{n+1} S^{1}=. A: y^{n} s^{1}$ so that $t_{1} y^{n} \in A$. Thus $a \in A$. Since the ideals $A \cup x S^{1}$ and $A \cup y^{n} S^{1}$ contain A properly A is not irreducible. This is a contradiction, and hence we conclude that every irreducible ideal is a primary ideal.

(vii) Let $A$ be an ideal of a noetherian semigroup $S$. In view of (vi) we can write $A=\bigcap_{i=1}^{n} Q_{i}$ where each $Q_{i}$ is a primary ideal. Clearly $\sqrt{Q}_{i}$ is a prime ideal, say $P_{i}$, for every i. Now $\sqrt{A}=\bigcap_{i=1}^{n} P_{i}$. Let $P$ be a minimal prime divisor of $A$. Then $\prod_{i=1}^{n} P_{i} \subseteq \bigcap_{i=1}^{n} P_{i}=\sqrt{A} \subseteq P$ so that $P_{i} \subseteq P$ for some $i$. We now have $A \subseteq P_{i} \subseteq P$ so that $P_{i}=P$ since $P$ is a minimal prime divisor of A. Thus every minimal prime divisor of $A$ is one of the $P_{i}^{\prime}$ 's occuring in the expression of $\sqrt{A}$. Hence $A$ has only a finite number of distinct minimal prime divisors. 
(viii) Let $A=\bigcup_{i=1}^{n} x_{i} s^{1}$ where $x_{i} k x_{j} s^{1}$ if $i \neq j$. Now $A=A^{2}=\bigcup_{i=1}^{n} x_{i} s^{1}=\bigcup_{i=1}^{n} x_{i}^{2} s^{1} \underset{j \neq k}{U} x_{j} x_{k} s^{1}$. It follows that $x_{i} \in x_{i}^{2} s^{1}$ so that $x_{i}=x_{i}^{2} s_{i}$ for some $s_{i} \in S$. Clearly $x_{i} s_{i}$ is an idempotent, say $e_{i}$. For every $i$, we have an idempotent $e_{i}=x_{i} s_{i}$. Now $x_{i}=x_{i}^{2} s_{i}=x_{i} e_{i} \in e_{i} s$ so that $x_{i} s^{1} \subseteq e_{i} s$. On the other hand $e_{i}=x_{i} s_{i} \in x_{i} s \subseteq x_{i} s^{l}$ so $e_{i} s \subseteq x_{i} s^{l}$. Thus $x_{i} s^{1}=e_{i} s$ for every $i$, which shows that $A=\bigcup_{i=1}^{n} e_{i} S$, where $e_{i}^{\prime} s$ are idempotents.

(ix) We can write $\left(\underset{\alpha}{U} \mathrm{P}_{\alpha}\right)^{2}=\underset{\alpha}{U} \mathrm{P}_{\alpha}^{2} \underset{\beta \neq \gamma}{\cup} \mathrm{P}_{\beta, \gamma} \mathrm{P}_{\gamma}=\underset{\alpha}{U} \mathrm{P}_{\alpha} \underset{\beta \neq \gamma}{U} \mathrm{P}_{\beta} \mathrm{P}_{\gamma}$, since each $P_{\alpha}$ is an idempotent ideal. clearly $\underset{\alpha}{U} P_{\alpha} \underset{\beta \neq \gamma}{U} P_{\beta} P_{\gamma}=\underset{\alpha}{U} P_{\alpha}$ and so $\bigcup_{\alpha} \mathrm{P}_{\alpha}$ is an idempotent ideal. Now suppose $\bigcup_{\alpha} \mathrm{P}_{\alpha}=$ as $^{1}$ for some $\mathrm{a} \in \mathrm{S}$. Then $\mathrm{a} \in \mathrm{P}_{\alpha}$ for some $\alpha$, so that $\mathrm{as}^{1} \subseteq \mathrm{P}_{\alpha}$, i.e., $\mathrm{as}^{1}=\mathrm{UP}_{\alpha} \subseteq \mathrm{P}_{\alpha}$. Thus $\mathrm{P}_{\alpha}=\mathrm{as}^{1}$, which is a contradiction. Hence $\cup P_{\alpha}$ is not a principal ideal.

$\alpha \quad(x)$ We can write $P A=\prod_{i=1}^{n} Q_{i}^{k}$ where $Q_{i}^{\prime}$ 's are all the distinct minimal prime divisors of the ideal PA. Clearly $P A \subseteq Q_{i}$ for every $i$ so that $P$ or $A$ is contained in $Q_{i}$ for every $i$. If $P \subseteq Q_{i}$ for some $i$, then $P A \subseteq P \subseteq Q_{i}$ so that $P=Q_{i}$. Suppose $A \subseteq Q_{i}$ for some $i$. Then $P A \subseteq P<A \subseteq Q_{i}$ which contradicts the fact that $Q_{i}$ is a minimal prime divisor of $P A$. Thus $Q_{i}=P$ for every $i$, in which case we have $P A=P^{k}$ for some positive integer $k$ and $P$ is the only minimal prime divisor of PA. Now $\mathrm{P}^{\mathrm{k}}$ is P-primary so that $\mathrm{P} \subseteq \mathrm{P}^{\mathrm{k}}$ since $\mathrm{A} \nsubseteq \mathrm{P}$. Thus $\mathrm{P}=\mathrm{P}^{\mathrm{k}}=\mathrm{PA}$.

(xi) Suppose $\mathrm{P}^{w} \neq \emptyset$. Let $\mathrm{x}, \mathrm{y} \notin \mathrm{P}^{(\omega)}$. If $\mathrm{x}, \mathrm{y} \in \mathrm{S} \backslash \mathrm{P}$ then 
$x y \notin \mathrm{P}$ so that $x y \in \mathrm{P}^{\omega}$. If $\mathrm{x} \in \mathrm{P}, \mathrm{y} \in \mathrm{S} \backslash \mathrm{P}$ then for some positive integer $r$ we have $x \in \mathrm{P}^{\mathrm{r}} \backslash \mathrm{P}^{\mathrm{r}+1}$. We show that $x y k \mathrm{P}^{\mathrm{r}+1}$. Suppose $x y \in \mathrm{P}^{\mathrm{r}+1}$. Since $\mathrm{p}^{\mathrm{r}+1}$ has radical $\mathrm{P}$, from hypothesis it follows that $\mathrm{P}^{\mathrm{r}+1}$ is $\mathrm{P}$-primary and so $\mathrm{x} \in \mathrm{P}^{\mathrm{r+1}}$ or $\mathrm{y} \in \mathrm{P}$, which is not possible. Thus $x y \in \mathrm{P}^{\mathrm{r}+1}$ and hence $x y \in \mathrm{P}^{\omega}$. Finally if $x, y \in \mathrm{P}$ then $x \in P^{r} \backslash P^{r+1}$ and $y \in P^{s} \backslash P^{s+1}$ for some positive integers $r$ and $s$. We note that $\sqrt{x s^{1} \cup P^{r+1}}=P=\sqrt{y s^{1} \cup p^{s+1}}$. Now $x s^{1} \cup P^{r+1}$ and $y s^{1} \cup \mathrm{p}^{\mathrm{s}+1}$ are $\mathrm{P}$-primary and so each is some power of $\mathrm{P}$. Clearly $x s^{1} \cup \mathrm{P}^{\mathrm{r+1}}=\mathrm{P}^{\mathrm{r}}$ and $y \mathrm{~s}^{1} \cup \mathrm{P}^{\mathrm{s}+1}=\mathrm{P}^{\mathrm{s}}$. We have then $\mathrm{p}^{\mathrm{r}+\mathrm{s}}=\mathrm{p}^{\mathrm{r}} \mathrm{P}^{\mathrm{s}}=\left(\mathrm{xs}^{1} \cup \mathrm{p}^{\mathrm{r}+1}\right)\left(y \mathrm{~s}^{1} \cup \mathrm{p}^{\mathrm{s}+1}\right) \subseteq x y \mathrm{~s}^{1} \cup\left(x \mathrm{~s}^{1}\right) \mathrm{p}^{\mathrm{s}+1} \cup\left(\mathrm{p}^{\mathrm{r}+1}\right)\left(y \mathrm{~s}^{1}\right)$ $\cup \mathrm{P}^{\mathrm{r}+\mathrm{s}+2} \subseteq \mathrm{xys}^{1} \cup \mathrm{P}^{\mathrm{r}+\mathrm{s}+1} \cup \mathrm{P}^{\mathrm{r}+\mathrm{s}+1} \cup \mathrm{P}^{\mathrm{r}+\mathrm{s}+2} \subseteq x y s^{1} \cup \mathrm{P}^{\mathrm{r}+\mathrm{s}+1}$. We claim that $x y \in \mathrm{p}^{\mathrm{r+s+1}}$. For, if $x y \in \mathrm{P}^{\mathrm{r}+\mathrm{s}+1}$ then it follows from the above that $\mathrm{P}^{\mathrm{r}+\mathrm{s}} \subseteq \mathrm{P}^{\mathrm{r}+\mathrm{s}+1}$, i.e., $\mathrm{P}^{\mathrm{r}+\mathrm{s}}=\mathrm{P}^{\mathrm{r+s}+1}$, which contradicts the fact that powers of $P$ properly descend. Therefore $x y \in \mathrm{P}^{\mathrm{r+s}+1}$ so that $x y \notin \mathrm{P}^{(1)}$. Hence $\mathrm{P}^{\omega}$ is a prime ideal with $\mathrm{P}^{(1)}<\mathrm{P}$. (xii) If $A \subseteq P^{n}$ and $A \nsubseteq \mathrm{P}^{\mathrm{n}+1}$ then $\mathrm{P}^{\mathrm{n}}$ is the isolated P-primary component of $A$, since every P-primary dideal containing $A$ is some power of $P$. Suppose that $A \subseteq P^{W}$. If powers of $P$ properly descend then $\mathrm{P}^{(\omega)}$ is a prime ideal by $(x i)$. This shows that $A \subseteq \mathrm{P}^{(\omega)}<\mathrm{P}$ which is a contradiction to the fact that $P$ is a minimal prime divisor of $A$. Hence $\mathrm{P}^{\mathrm{m}}=\mathrm{P}^{\mathrm{m}+1}$ for some natural number $\mathrm{m}$, if $A \subseteq \mathrm{P}^{\mathrm{w}}$. If $\mathrm{n}$ is the least positive integer such that $\mathrm{P}^{\mathrm{n}}=\mathrm{P}^{\mathrm{n}+1}$ then $\mathrm{P}^{\mathrm{n}}$ is the isolated P-primary component of A.

(xiii) Let $A$ be an ideal of $S$ with $\sqrt{A}=P$, where $P$ is a prime ideal. Clearly $P$ is the only minimal prime divisor of $A$. Since $A=\operatorname{Ker} A$, we have $A=\bigcap_{\alpha} Q_{\alpha}$ where each $Q_{\alpha}$ is a P-primary 
ideal containing $A$. We show that $A$ is a P-primary ideal. Let $x y \in A$ with $x \notin A$. Then $x \notin Q_{\alpha}$ for some $\alpha$. Clearly $x y \in Q_{\alpha}$ with $x \notin Q_{\alpha}$ so that $y \in \sqrt{Q}_{\alpha}=P$ since $Q_{\alpha}$ is a P-primary ideal. Thus we have $y \in P=\sqrt{A}$ which shows that $A$ is a P-primary ideal. (xiv) We may assume that $S$ does not contain a unique maximal ideal since otherwise the result is trivial. Let $M$ be a maximal ideal of $S$ such that $M \neq M^{2}$. Clearly $M^{2} U M^{*} \subseteq M$. Now let $x \in M$. If $x \notin M^{*}$ then $x \notin P$ for some maximal ideal $P$ of $S$. Clearly $\mathrm{x}^{2} \notin \mathrm{P}$ since $\mathrm{S}=\mathrm{S}^{2}$ implies that $\mathrm{P}$ is a prime ideal of $\mathrm{S}$ [18]. Now $\mathrm{P} \cup \mathrm{x}^{2} \mathrm{~s}^{1}=\mathrm{S}$ and so $\mathrm{x}=\mathrm{x}^{2} \mathrm{~s}$ for some $\mathrm{s} \in \mathrm{S}$ since $\mathrm{x} \notin \mathrm{P}$. Thus $x=x^{2} s \in M^{2}$ and so $M \subseteq M^{2} \cup M^{*}$. Hence $M=M^{2} \cup M^{*}$.

NOTE: The following example shows that the converse of anyone of (iv), (vi), and (vii) in the above Proposition need not be true. For, $S=\left\{x_{1}, x_{2}, \ldots\right\}$ with $x_{i} x_{j}=x_{\min (i, j)}$ is a semigroup in which the set of ideals is linearly ordered. In fact every ideal of $S$ is a prime ideal. However, $S$ considered as an ideal is not principal; actually it is not even finitely generated.

2.15 NOTATION. We denote by $\mathrm{z}$ the set of all non-cancellable elements of $s$, which can be easily seen to be a prime ideal of $s$.

\subsection{PROPERTIES OF COMMUTATIVE MULTIPLICATION SEMIGROUPS}

We recall that a multiplication semigroup is a semigroup in which for any pair of ideals $A$ and $B$ with $A \subseteq B$ we have $A=C B$ for some ideal $\mathrm{C}$ of $\mathrm{S}$. 
2.16 THEOREM. In a multiplication semigroup $\mathrm{S}$, the following are true.

(i) $\mathrm{S}=\mathrm{S}^{2}, \mathrm{~A}=\mathrm{AS}$ for any ideal $\mathrm{A}, \mathrm{aS}^{1}=\mathrm{aS}$ for every $a \in S$.

(ii) If $\mathrm{S}$ contains a cancellable element then $\mathrm{S}$ contains identity.

(iii) If $\mathrm{P}$ is a prime ideal which is properly contained in an ideal $A$ then $\mathrm{P}=\mathrm{PA}$; moreover, $\mathrm{P}=\mathrm{A}^{\omega}$ or $\mathrm{P}=\mathrm{PA}$.

(iv) If $M$ is a maximal ideal of $S$ then there are no ideals between $M^{n}$ and $M^{n+1}$ for any natural number $n$.. Consequently, if $M^{n} \neq M^{n+1}$ then $M^{n}=x s^{1}$ or $M^{n}=M^{n+1} \cup x s^{1}$ for some $x \in M^{n} \vee M^{n+1}$.

(v) Every ideal with prime radical is primary. In particular, if $P$ is a prime ideal then $P^{n}$ is P-primary for any natural number $n$.

(vi) If $P$ is a prime ideal with powers of $P$ properly descending then $P^{W}$ is either empty or is a prime ideal with $P^{(y)}<P$.

(vii) Every primary ideal is some power of its radical.

(viii) An ideal is a primary ideal if and only if it is some power of its prime radical.

(ix) If $P$ is a prime ideal and $A$ is any ideal such that $A \subseteq P^{n}, A \nsubseteq P^{n+1}$ for some natural number $n$ then $P^{n}=A: y S$ for some $y \in S \backslash P$.

(x) If $A$ is an idempotent ideal of $S$ then $A$ is a multiplication semigroup; in particular, for any $x \in A$ we have $x=x y$ for some $y \in A$.

(xi) Every homomorphic image of $\mathrm{S}$ is a multiplication semigroup; also $\mathrm{s}^{1}$ is a multiplication semigroup. 
(xii) Every proper ideal is contained in a proper prime ideal; every proper ideal of $S$ has a proper minimal prime divisor and hence $S$ is not archimedian.

(xiii) If $\mathrm{P}$ is a proper prime ideal of $\mathrm{S}$ which is not contained in any proper prime ideal then $P$ is a maximal ideal.

(xiv) Suppose $\mathrm{P}^{\mathbb{W}}=\emptyset$ for every proper prime ideal $\mathrm{P}$ of $\mathrm{S}$. If $L$ and $M$ are any two proper ideals of $S$ with $L<M$ then there exists a proper prime ideal $\mathrm{P}$ such that $\mathrm{L} \subseteq \mathrm{P}^{\mathrm{n}}$ and $\mathrm{M} \nsubseteq \mathrm{P}^{\mathrm{n}}$ for some natural number $\mathbf{n}$.

(xv) If $\mathrm{P}$ is, a non-idempotent prime ideal then $\mathrm{P}$ contains at most one prime ideal $Q$ with no prime ideals between $P$ and $Q$.

(xvi) If $P$ is a principal prime ideal then $P$ contains at most one prime ideal $Q$ with no prime ideals between $P$ and $Q$.

(xvii) If the set of prime ideals of $S$ is linearly ordered then every non-idempotent prime ideal is principal.

(xviii) For any finite set of distinct prime ideals $P_{1}, \ldots, P_{n}$ we have $\prod_{i=1}^{n} P_{i}^{k_{i}}=\bigcap_{i=1}^{n} P_{i}^{k_{i}}$ where $k_{i}^{\prime}$ 's are some positive integers.

(xix) If $S$ is noetherian, then every ideal has a finite number of distinct minimal prime divisors and is a product of its minimal prime divisors.

(xx) If $S$ is noetherian then $A=\operatorname{Ker} A$ for every ideal $A$ of $S$.

(xxi) If $\operatorname{dim} S<\infty$ then $S$ contains maximal ideals.

(xxii) If $\operatorname{dim} S^{1} \leq 1$ then $A=\operatorname{Ker} A$ for every ideal $A$ of $S$; In particular, if prime ideals of $S$ are maximal then $A=\operatorname{Ker} A$ for every ideal $A$ of $S$. 
(xxiii) For any maximal ideal $M$ of $S$ we have $M=M^{2}$ or $M=M^{2} U M^{*}$ where $M^{*}$ is the intersection of all maximal ideals of $S$. (xxiv) For every $x, y \in S$ we have $x \in y s^{1}$ or $y \in x s^{1}$ or $x y \in x^{2} s^{1} \cap y^{2} s^{l}$.

$(x \times v) s^{1}$ is a multiplication semigroup with $s=s^{2}$.

(xxvi) $S$ contains identity if and only if $S$ contains a unique maximal ideal $M$ such that every proper ideal is contained in $M$.

(xxvii) If $S$ contains identity and if $M$ is the unique maximal ideal of $S$ then for every proper ideal $A$ of $S$ we have either $A=M^{n}$ for some natural number $n$ where $M^{n} \neq M^{n+1}$ or $A \subseteq M^{W}$; in particular, if $M^{\omega}=\emptyset$ then every proper ideal of $S$ is some power of $M$.

(xxviii) If $x$ and $y$ are cancellable and non-cancellable elements respectively, then $y S<x S$; consequently $z<x S$.

(xxix) If $S$ contains cancellable non-units then for some cancellable element $x, x S$ is the unique maximal ideal and $z=(x S)^{4}$.

$(x x x) \quad z=z^{2}$ or $z=y S$ for some non-idempotent element $y$ in S.

Proof. (i) and (ii) are proved in 1.16 .

From now on we use the property $a s^{1}=a s$ for every $a \in S$ without reference.

(iii) Since $P<A$ we have $P=B A$ for some ideal $B$, so that $\mathrm{B} \subseteq \mathrm{P}$ since $\mathrm{P}$ is a prime ideal with $\mathrm{A} \nsubseteq \mathrm{P}$. Now $\mathrm{P}=\mathrm{BA} \subseteq \mathrm{PA} \subseteq \mathrm{P}$ so that $\mathrm{P}=\mathrm{PA}$. It then follows that $\mathrm{P} \subseteq A^{W}$. If $P \neq A^{W}$ then $P<A^{\omega}$ and so from the above we have $\mathrm{P}=\mathrm{PA}^{\mathrm{W}}$. 
(iv) Let $A$ be an ideal of $S$ such that $M^{n+1}<A<M^{n}$ for some natural number $n$. Now $A=M^{n} B$ for some ideal $B$ where $B \nsubseteq M$. Let $x \in B \backslash M$. Since $M U x S=S$ we have now $M^{n}=M^{n} S=M^{n}(M \cup x S)=M^{n+1} \cup M^{n}(x S) \subseteq A$, which is a contradiction. Suppose $M^{n} \neq M^{n+1}$. Let $x \in M^{n} \backslash M^{n+1}$. If $M^{n+1}<x s^{1}$ then from the above it follows that $M^{n}=x S^{1}$. If $M^{n+1}$ is not contained in $x S^{1}$ then $M^{n}=M^{n+1} \cup x S^{1}$ by a similar argument.

(v) Let $Q$ be an ideal with $\sqrt{Q}=P$, where $P$ is a prime ideal. If $\mathrm{P}=\mathrm{S}$, clearly $\mathrm{Q}$ is primary. We may now assume $\mathrm{P} \neq \mathrm{S}$. Suppose $Q$ is not primary. Then there exist $p \in P \backslash Q$ and $r \in S \backslash P$ such that preQ. We set $A=Q U(\mathrm{pS}) \mathrm{P}$. We first show that $p \notin A$. If $p \in A$ then $p=p p^{\prime}$ for some $p^{\prime} \in P=\sqrt{Q}$. Now $p^{, n} \in Q$ for some positive integer $n$ so that $p=p p^{1 n} \in Q$ which leads to a contradiction. Therefore $p \notin A$. Since $A \cup p S \subseteq P$ we have $A \cup p S=P L$ for some ideal L. We also have $P=P(P \cup r S)$ in view of (iii) since $\mathrm{P}<\mathrm{P} \cup \mathrm{rS}$. It follows that $\mathrm{A} \cup \mathrm{pS}=\mathrm{P}(\mathrm{P} \cup \mathrm{rS}) \mathrm{L}=(\mathrm{A} \cup \mathrm{pS})(\mathrm{P} \cup \mathrm{rS}) \subseteq \mathrm{A}$, i.e., $p \in A$, which is a contradiction. Hence $Q$ is a primary ideal. Now if $\mathrm{P}$ is a prime ideal then $\mathrm{P}^{\mathrm{n}}$ has radical $\mathrm{P}$ for any natural number $n$ so that from the above it follows that $p^{n}$ is $P$-primary. (vi) Suppose that $P^{\#)} \neq \varnothing$. Let $A$ and $B$ be any two ideals with $A B \subseteq P^{\omega}, A \nsubseteq P^{\omega}$ and $B \nsubseteq P^{\omega}$. Now $A B \subseteq P^{\omega} \subseteq P$ so that $A \subseteq P$ or $\mathrm{B} \subseteq \mathrm{P}$ since $\mathrm{P}$ is a prime ideal. We have the following possibilities: $A \nsubseteq P, B \subseteq P$ or $A \subseteq P, B \nsubseteq P$ or $A, B \subseteq P$. In the first case, there exists a natural number $k$ such that $B \subseteq \mathrm{P}^{\mathrm{k}}, \mathrm{B} \nsubseteq \mathrm{P}^{\mathrm{k}+1}$. Now by the multiplication property we have $B=\mathrm{P}^{\mathrm{k}} \mathrm{C}$ for some ideal $\mathrm{C}$ with $C \nsubseteq \mathrm{P}$. Now $\mathrm{AB}=\mathrm{ACP}^{\mathrm{k}} \subseteq \mathrm{P}^{w}$ so that $\mathrm{ACP}^{\mathrm{k}} \subseteq \mathrm{P}^{\mathrm{k}+1}$. But $\mathrm{P}^{\mathrm{k}+1}$ is $\mathrm{P}$-primary 
by $(v)$ so that $\mathrm{P}^{\mathrm{k}} \subseteq \mathrm{P}^{\mathrm{k}+1}$ since AC $\nsubseteq \mathrm{P}$. Thus $\mathrm{p}^{\mathrm{k}}=\mathrm{P}^{\mathrm{k}+1}$ which is a contradiction since powers of $\mathrm{P}$ properly descend. By a similar argument we get a contradiction in the second case also. Finally if $A, B \subseteq P$ then for some natural numbers $m$ and $n$ we have $A \subseteq P^{m}$, $A \nsubseteq p^{m+1}$ and $B \subseteq P^{n}, B \nsubseteq P^{n+1}$. Now by the multiplication property $A=P_{D}^{m_{D}}$ and $B=P^{n_{L}}$ for some ideals $D$ and $L$ where $D, L \nsubseteq P$. Now $A B=\mathrm{p}^{\mathrm{m}+\mathrm{n}} \mathrm{DL}$ where $\mathrm{DL} \nsubseteq \mathrm{P}$. But $A B \subseteq \mathrm{P}^{\omega}$ so that $\mathrm{P}^{\mathrm{m}+\mathrm{n}} \mathrm{DL} \subseteq \mathrm{P}^{\mathrm{m}+\mathrm{n}+1}$. By (v) $\mathrm{P}^{\mathrm{m}+\mathrm{n}+1}$ is $\mathrm{P}$-primary so that $\mathrm{p}^{\mathrm{m}+\mathrm{n}} \subseteq \mathrm{p}^{\mathrm{m}+\mathrm{n}+1}$ since DL $\nsubseteq \mathrm{P}$. Thus we have $P^{m+n}=P^{m+n+1}$, which is again a contradiction to the fact that powers of $\mathrm{P}$ properly descend. Therefore we must have $\mathrm{A}$ or $\mathrm{B} \subseteq \mathrm{P}^{\omega}$, which shows that $\mathrm{P}^{\omega}$ is a prime ideal. Clearly $\mathrm{P}^{W}<\mathrm{P}$. (vii) Let $Q$ be a primary ideal with $\sqrt{Q}=P$. If $P=S$ then $Q=s$. For, if $x \in S$ then $x=x s$ for some $s \in S=\sqrt{Q}$. Now $s^{k} \in Q$ for some natural number $k$ so that $x=x s^{k} \in Q$. Thus $Q=S$. We may now assume that $P \neq S$. Suppose $Q \subseteq P^{w}$. If $P^{n} \neq P^{n+1}$ for any natural number $n$ then by (vi), $P^{w}$ is a prime ideal. This shows that $\mathrm{P}=\sqrt{\mathrm{Q}} \subseteq \sqrt{\overline{\mathrm{P}}^{-\omega}}=\mathrm{P}^{\omega}$, which is a contradiction. Hence we have the possibilities: either $Q \subseteq \mathrm{P}^{\omega}$ with $\mathrm{P}^{\mathrm{n}}=\mathrm{P}^{\mathrm{n}+1}$ for some natural. number $n$ or $Q \subseteq p^{n}, Q \nsubseteq p^{n+1}$ for some natural number $n$. In the first case, for any $x \in P^{n}$ we have $x S=P^{n} A$ for some ideal $A$. Then $x S=P_{A} n^{n+1} A=P(x S)=x P$ so that $x=x t$ for some $t \in P=\sqrt{Q}$. Since $t^{k} \in Q$ for some natural number $k$, we have $x=x t^{k} \in Q$. Hence $P^{n}=Q$. In the second case, we have $Q=P^{n} C$ for some ideal $C$ where $C \nsubseteq P$. Since $Q$ is P-primary, clearly $P^{n} \subseteq Q$ and thus $Q=\mathrm{P}^{\mathrm{n}}$.

Here we note that every ideal with a unique minimal prime divisor 
$P$ is some power of $P$ in view of (v) and (vii). We use this fact often in our proofs.

(viii) An application of (v) and (vii) yields this result.

(ix) We can write $A=P^{n_{B}}$ for some ideal $B$ where $B \nsubseteq P$. Let $y \in B \backslash P$. Then $P^{n}(y S) \subseteq A$, i.e., $P^{n} \subseteq A: y S \vdots$. Now let $x \in A: y S$. Then $(x S)(y S) \subseteq A \subseteq P^{n}$. By (v) $P^{n}$ is P-primary so that $x S \subseteq P^{n}$ since $y S \nsubseteq P$. Thus we have $x \in \mathrm{P}^{n}$ which shows that $A: y S \subseteq P^{n}$. Hence $\mathrm{P}^{\mathrm{n}}=\mathrm{A}: \mathrm{yS}$.

(x) Let $B$ be an ideal of $A$. Then $B S=A C$ for some ideal $C$ of $S$ since $B S \subseteq A S=A$. Now we have $B S=A C=A^{2} C=A B S=B(A S)=$ $=B A \subseteq B$ and thus $B$ is an ideal of $S$. Now $B \subseteq A$ where $B$ and $A$ are ideals of $S$ so that $B=A D$ for some ideal $D$ of $S$. Thus we have $B=A D=A^{2} D=A B$. Now for any ideal $B$ of $A$ we have $B=A B$. Let $B_{1}$ and $B_{2}$ be any two ideals of $A$ with $B_{1} \subseteq B_{2}$. Then $B_{1}$ and $B_{2}$ are also ideals of $S$ so that $B_{1}=B_{2} L$ for some ideal $L$ of S. Since $B_{2}=B_{2} A$ we have $B_{1}=B_{2} A L$. Now $A L$ is an ideal of $A$. Hence $A$ is a multiplication semigroup. Finally for any $x \in A$, $x \cup x A$ is an ideal of $A$ so that $x \cup x A=(x \cup \times A) A=x A$. Clearly $x=x y$ for some $y \in A$.

(xi) A direct verification yeilds the result.

(xii) If $A$ is a proper ideal of $S$ which is not contained in any proper prime ideal, then clearly $S$ is the only minimal prime divisor of $A$ so that $A=S^{n}$ for some natural number $n$ and thus $A=: S$ which is not true. Thus $A$ is contained in a proper prime ideal, say P. Now by (ii) of Theorem 2.14, P contains a minimal prime divisor of $A$, which is evidently a proper minimal prime divisor 
of A. Since it is assumed that $S$ is not a group, $S$ contains proper ideals and hence proper prime ideals. Thus $\mathrm{S}$ is not archimedian [12].

(xiii) Let $P$ be a proper prime ideal which is not contained in any proper prine ideal. Suppose that $P$ is not maximal. Then there exists a proper ideal A with $P<A$. Clearly $A$ is not contained in any proper prime ideal. But this is not possible in view of (xii). Hence $P$ is a maximal ideal.

(xiv) Pick $d \in M \backslash L$. We can write $d=d y$ for some $y \in S$ and so $\mathrm{d}=\mathrm{dy}=\mathrm{dy} \mathrm{y}^{2}$ etc. This implies that $y^{\mathrm{n}} \ell \cdot \mathrm{L}$ : dS for any natural number $n$. Now $L ; d S$; : being a proper ideal of $S$, has a proper minimal prime divisor $P$ by (xii). We claim that $L=(L: M) M$. Trivially $(L: M) M \subseteq L$. Since $L \subseteq M$ we have $L=M B$ for some ideal $B$ of $S$, i.e., $B \subseteq L: M$. Thus we have $L \subseteq(L: M) M$ so that $L=(L: M) M$. It then follows that $(L: M) M \subseteq(L: d S) M \subseteq P M$, i.e., $L \subseteq P M$. If $M \subseteq \mathrm{P}^{\mathrm{n}}$ for all natural numbers $\mathrm{n}$ then $\mathrm{L}<M=\emptyset$ which is not true. Thus for some natural number $n>1$, either $M \subseteq P^{n-1}$ and $M \nsubseteq P^{n}$ or $M \nsubseteq P$. In the first case we have $L \subseteq \mathrm{P}^{n}$ and $M \nsubseteq \mathrm{P}^{\mathrm{n}}$ while in the latter case $L \subseteq P$ and $M \nsubseteq P$.

(xv) Suppose $Q$ and $Q^{\prime}$ are two distinct prime ideals such that $Q<P$ and $Q^{\prime}<P$ with no prime ideals between $Q$ and $P$, and $Q^{\prime}$ and $P$. Since $Q<Q \cup Q^{\prime} \subseteq P$, we have $Q \cup Q^{\prime}=P$. By (iii) $Q=Q P$ and $Q^{\prime}=Q^{\prime} P$ so that $Q P \cup Q^{\prime} P=Q \cup Q^{\prime}=P^{2}$, i.e., $P=P^{2}$ which is a contradiction.

(xvi) If possible, let $Q$ and $Q^{\prime}$ be as in the above, where $P$ is a principal prime ideal. Now $Q \cup Q^{\prime}=P=x S$ so that $x \in Q$ or $Q^{\prime}$. It follows that $\mathrm{P}=\mathrm{xS} \subseteq \mathrm{Q}$ or $\mathrm{Q}^{\prime}$, which is a contradiction. 
(xvii) Let $\mathrm{P}$ be a non-idempotent prime ideal. For any $\mathrm{x} \in \mathrm{P} \backslash \mathrm{P}^{2}$, $P$ is the only minimal prime divisor of the ideal $x S$, since prime ideals are linearly ordered. Now $x S=P^{r}$ and thus $x S=P$.

(xviii) Since $\bigcap_{i=1}^{n} P_{i}^{k_{i}} \subseteq P_{1}{ }_{1}$ we have $\bigcap_{i=1}^{n} P_{i}^{k_{i}}=P_{1}^{k_{1}} A_{1}$ for some ideal $A_{1}$. Now $P_{1}^{{ }^{k}} A_{1}=\bigcap_{i=1}^{n} P_{i}^{k} \subseteq P_{2}{ }^{k}$. By (v) $P_{2}^{{ }^{2}}$ is $P_{2}$-primary so $\mathrm{P}_{1}^{\mathrm{k}_{1}} \subseteq \mathrm{P}_{2}$ or $\mathrm{A}_{1} \subseteq \mathrm{P}_{2}^{\mathrm{k}_{2}}$. If $\mathrm{P}_{1}^{\mathrm{k}_{1}} \subseteq \mathrm{P}_{2}$ then $\mathrm{P}_{1}<\mathrm{P}_{2}$ so by (iii) we have $P_{1}=P_{1} P_{2}=P_{1} P_{2}^{2}=\ldots=P_{1} P_{2}^{k}$ and thus $P_{1}^{k_{1}}=P_{1}^{k_{1}} P_{2}^{k_{2}}$. In this case we have $\bigcap_{i=1}^{n} P_{i}^{k_{i}}=P_{1}^{k} P_{2}^{k_{2}} A_{1}$. On the other hand, if $A_{1} \subseteq P_{2}^{k_{2}}$ then $A_{1}=P_{2}^{k_{2}} A_{2}$ so $\bigcap_{i=1}^{n} P_{i}^{k}=P_{1}^{k} P_{2}^{k_{2}} A_{2}$. Proceeding we obtain $\bigcap_{i=1}^{n} P_{i}^{k_{i}}={ }_{i=1}^{n} P_{i}^{k_{i}} A$ for some ideal $A$, from which it follows that $\prod_{i=1}^{n} P_{i}^{k}=\bigcap_{i=1}^{n} P_{i}{ }_{i}$

(xix) Since $S$ is a noetherian semigroup, for any ideal $A$ of $S$ in view of (vi) of Theorem 2.14 we can write $A=\bigcap_{i=1}^{n} Q_{i}$ where each $Q_{i}$ is a primary ideal. By (viii) we have $Q_{i}=P_{i} k_{i}$ for some natural number $k_{i}$ where $P_{i}=\sqrt{Q}_{i}$, so that $A=\bigcap_{i=1}^{n} P_{i}^{k_{i}}$. In fact we can write $A=\bigcap_{i=1}^{n} P_{i}^{k_{i}}$ with $P_{i} \neq P_{j}$. By (xviii) we have $A=\bigcap_{i=1}^{n} P_{i}^{k_{i}}=\prod_{i=1}^{n} P_{i}^{k_{i}}$. If $P_{i}<P_{j}$ for some $i \neq j$ then by (iii) $P_{i}=P_{i} P_{j}=\ldots=P_{i} P_{j}^{k}$ so that $P_{i}^{k_{i}}=P_{i}^{k_{i}} P_{j}^{k_{j}}$. We omit the factors $P_{j}^{k}$ if $P_{j}$ properly contains $P_{i}$ for some $i \neq j$ and write $A=\prod_{i=1}^{n} P_{i}^{k}$ 
where $P_{i} \notin P_{j}$ for $i \neq j$. Now we claim that the $P_{i}^{\prime}$ 's occuring in the product expression of $A$ are precisely the minimal prime divisors of A. We first show that each $P_{i}$ is a minimal prime divisor of $A$. For some $i$ if $P_{i}$ is not a minimal prime divisor of $A$ then $P_{i}$ properly contains a minimal prime divisor $Q$ of $A$. But then

$\ddot{A}=\prod_{i=1}^{n} P_{i}^{k} \subseteq Q<P_{i}$ so that $Q$ ccontains some $P_{j}$, i.e., $P_{j} \subseteq Q<P_{i}$ which is not possible. Therefore each $P_{i}$ is a minimal prime divisor of A. Now, if $Q$ is a minimal prime divisor of $A$ then $A=\prod_{i=1}^{n} P_{i}^{k} \subseteq Q$ so $Q$ contains some $P_{i}$, i.e., $A \subseteq P_{i} \subseteq Q$ for some i. But $P_{i}$ is a minimal prime divisor of $A$ so $P_{i}=Q$. Thus $A=\prod_{i=1}^{n} P_{i}^{k_{i}}$ where $P_{1}, \ldots, P_{n}$ are precisely the minimal prime divisors of $A$.

$((x x)$ If $A$ is an ideal of $S$ then by ( $x i x)$ A has only a finite number of distinct minimal prime divisors, say $\mathrm{P}_{1}, \mathrm{P}_{2}, \ldots, \mathrm{P}_{\mathrm{n}}$ and $A=\prod_{i=1}^{n} P_{i}^{k_{i}}$ for some natural numbers $k_{i} \cdot$ By (xviii) we have $A=\prod_{i=1}^{n} P_{i}^{k_{i}}=\bigcap_{i=1}^{n} P_{i}^{k_{i}}$. For every $i$, we may choose $t_{i}$ such that $P_{i}^{t_{i}}$ is the isolated $P_{i}$-primary component of $A$ in view of (xii) of Theorem 2.14, (v) and (vii). Now we can write $\operatorname{Ker} A=\bigcap_{i=1}^{n} p_{i}{ }_{i}$. We show that $P_{i}^{t_{i}}=P_{i}^{k_{i}}, \quad 1 \leq i \leq n$, so that we obtain $A=$ Ker A. Clearly $\prod_{i=1}^{n} P_{i}^{k_{i}} \subseteq \bigcap_{i=1}^{n} P_{i}^{k_{i}}=A \subseteq \operatorname{Ker} A=\bigcap_{i=1}^{n} P_{i}^{t_{i}} \subseteq P_{i}^{t_{i}}$ for every $i$. Now every $P_{i}^{t_{i}}$ is $P_{i}$-primary by $(v)$ and $P_{j} \nsubseteq P_{i}$ for $i \neq j$ so that $P_{i}^{k_{i}} \subseteq P_{i}^{{ }^{t}}$. 
But $P_{i}^{k_{i}}$ being $P_{i}$-primary ideal containing $A$, we have ${ }_{P_{i}}^{{ }^{t}} \subseteq P_{i}{ }_{i}$ since $P_{i}^{{ }^{t}}{ }_{i}$ is the isolated $P_{i}$-primary component of $A$. Thus $P_{i}^{k_{i}}=P_{i}^{t}$. for every i, which completes the proof.

(xxi) Let $\operatorname{dim} S=n$ for some natural number $n$. Then there exists a chain of proper prime ideals of length $n: P_{0}<P_{1}<\ldots<P_{n}$ where $P_{n}$ is not contained in any proper prime ideal of $S$. Now $P_{n}$ is a maximal ideal of $\mathrm{S}$ by (xiii). Thus $\mathrm{S}$ contains maximal ideals.

(xxii) We note that $s^{l}$ contains a unique maximal ideal by (i) of Theorem 2:14. Let $A$ be any ideal of $S$. Then $A$ is an ideal of $S^{1}$ also. Suppose $A \neq \operatorname{Ker} A$ in $s^{1}$. Let $a \in \operatorname{Ker} A \backslash A$. Set $B=A:$ as. Let $P$ be a minimal prime divisor of $B$. Clearly $A \subseteq B \subseteq P$ and so $P$ contains a minimal prime divisor $Q$ of $A$ by (ii) of theorem 2.14. Suppose $Q=P$. Now $\mathrm{AS}_{Q}^{1} \cap \mathrm{S}^{1}$ is an ideal of $\mathrm{S}^{1}$ with radical $Q$ and $\mathrm{S}^{1}$ is a multiplication semigroup by ( $\left.x i\right)$ and so by (v) $A_{Q}^{1} \cap s^{1}$ is Q-primary. Then a $\in A S_{Q}^{1} \cap s^{1}$. We can write $a=a / 1=b / x$ where $b \in A$ and $x \in S^{1} \backslash Q$ is a cancellable element. Clearly $a x=b \cdot l \in A$, i.e., $a x \in A$ with $x \in S^{1} \backslash Q$. Thus $x \in A:$ aS $=B \subseteq Q$, which is a contradiction. Therefore we must have $Q<P$. Now if $S^{1}$ is zero-dimensional then $Q=P$ which is impossible, and thus $A=\operatorname{Ker} A$. On the other hand, if $\operatorname{dim} S^{1}=1$ then $P$ is the unique maximal ideal of $\mathrm{s}^{l}$. Now we have $B=\mathrm{P}^{k}$ for some natural number $k$, since $p$ is the only minimal prime divisor of $B$. Since $a \in Q$, we have aS $=Q C$ for some ideal $C$; moreover, $Q=Q P^{k}$ in view of (iii) since $Q<P$. Thus $a S=Q C=Q P^{k} C=P^{k}(a S)=B(a S) \subseteq A$, i.e., $a \in A$, which is a contradiction. Hence $A=\operatorname{Ker} A$ in $S^{1}$. In view of (v) of Theorem 2.14, $A=\operatorname{Ker} A$ in $S$ too. 
Suppose prime ideals of $s$ are maximal. Now $\operatorname{din} s^{1}=0$ or 1 according as $S$ contains identity or not. Now the result follows from the above part.

(xxiii) By (i), $s=s^{2}$ and so the proof follows from (xiv) of Theorem 2.14 .

(xxiv) Let $x$ and $y$ in $s$ be such that $x \notin y s^{l}$ and $y \notin x s^{l}$. Since $x s^{1} \subseteq x S^{1} \cup y s^{1}$ we have $x s^{1}=\left(x s^{1} \cup y s^{1}\right) A$ for some ideal $A$ of $s$. Now $x=x a$ for some $a \in A$ and ya $=x t$ for some $t \in S$. Then $x y a=x^{2} t$ and thus $x y=x^{2} t$. Since $y s^{1} \subseteq x s^{1} \cup y s^{1}$ it follows by a similar argunent as before that $x y=y^{2} s$ for some $s \in S$. Thus $x y \in x^{2} s^{1} \cup y^{2} s^{1}$.

(xxv) Evident in view of ( $v$ ) of Theorem 2.14.

In fact the converse is also true in view of $(x)$.

(xxvi) Suppose that $S$ contains a unique maximal ideal $M$ such that every proper ideal is contained in it. Let $x \in S \backslash M$. Then $S$ is the only minimal prime divisor of $x S$ so that $x S=s^{r}=s$. Also $x S=x^{2} s$ since $s=s^{2}$ by $(i)$, and so $s$ contains an identity. The other implication -is (evident by -(i) of Theorem 2.14.

(xxvii) The result follows from (ix).

(xxviii) In view of (ii) $\mathrm{S}$ contains identity. We may assume that $x S \nsubseteq y S$, for otherwise it follows that $y$ is a cancellable element, which is not true. Now $x S \subseteq x S U$ yS so that $x S=(x S \cup y S) A$ for some ideal $A$. Then $x=x s$ or $y t$ for some $s, t \in S$. clearly the latter is not possible by our assumption. We now have $x=x s$ which implies that $s$ is an identity in $S$. Thus $A=S$ and hence $y S \subseteq x S$. If $y S=x S$ then $x=y a$ for some $a \in S$ which is inadmissible. 
Thus we have $y S<x S$ which in turn shows that $z<x S$.

(xxix) In view of (ii) S contains identity and so by (i) of Theorem $2.14 \mathrm{~S}$ contains a unique maximal ideal $M$. If $M=M^{2}$ then by ( $x$ ) for any cancellable $x \in M, x=x y$ for some $y \in M$ which shows that $y$ is an identity of $S$. Now $M=S$ which is not true. Hence $M \neq M^{2}$. If $x \in M \backslash M^{2}$ then $x$ is a cancellable element. For otherwise $x \in Z<M$, where $Z \subseteq M^{2}$ by (iii), which contradicts the fact that $x \notin M^{2}$. Thus $x$ is a cancellable element and the only minimal prime divisor of $x S$ is $M$. Hence $x S=M$. We claim that $Z=M^{W}$. For this we note that powers of $M$ properly descend. For, if $M^{r}=M^{r+1}$ for some positive integer $r$ then $x^{r}=x^{r+1} s$ for some $s \in s$ which shows that $x$ is a unit, which is not so. Now by (vi) $M^{W}$ is a prime ideal. We have by (iii) $\mathrm{Z} \subseteq \mathrm{M}^{\omega}$. We now show that $\mathrm{M}^{\omega} \subseteq \mathrm{Z}$. Suppose that $M^{\omega}=\left(M^{\omega}\right)^{2}$. Then for any $y \in M^{\omega}, y=y s$ for some $s \in M^{\omega}$ by $(x)$. If $y \notin z$ then $s$ is an identity in $s$, which is not true. Thus $\mathrm{M}^{\omega} \subseteq \mathrm{Z}$ in this case. If $\mathrm{M}^{\omega} \neq\left(\mathrm{M}^{\omega}\right)^{2}$ then for any $\mathrm{y} \in \mathrm{M}^{\omega} \backslash\left(\mathrm{M}^{\omega}\right)^{2}$, $M^{\omega}$ is the only minimal prime divisor of $y S$ in view of (iii), so that yS $=M^{\omega}$. Now by (iii) $M^{\omega}=M^{\omega} M$ since $M^{\omega}$ is a prime ideal with $M^{\omega}<M$. We now have $y=y x t$ for some $t \in S$. If $y \notin z$ then $x$ is a unit, which is not so. Therefore $M^{\omega} \subseteq Z$. Hence $z=M^{\omega}=(x S)^{\omega}$. $(x \times x)$ If $s=z$ then by (i) $z=z^{2}$. Suppose $s \neq z$. Then by (ii) $S$ contains an identity and thus $S$ contains a unique maximal ideal $M$. If $M=Z$ and if $z \neq z^{2}$ then for any $y \in Z \backslash z^{2}, Z$ is the only minimal prime divisor of ys so that $\mathrm{Z}=\mathrm{yS}$. Suppose $\mathrm{Z} \neq \mathrm{M}$. If $P$ is a prime ideal such that $P<M$ then by (iii) and ( $x$ xix) $\mathrm{P} \subseteq \mathrm{z}$. If $\mathrm{z} \neq \mathrm{z}^{2}$, it then follows that for any $\mathrm{y} \in \mathrm{Z}$, that $\mathrm{Z}$ is the 
only minimal prime divisor of $y S$ so that we have $z=y S$. clearly $y \neq y^{2}$.

\subsection{COMMUTATIVE NOETHERIAN SEMIGROUPS}

We first note that principal ideal semigroups containing identity are multiplication semigroups. As we noted earlier, regular semigroups are also multiplication semigroups. However, these two classes do not exhaust the class of all multiplication semigroups. In this section we determine the conditions for a noetherian multiplication semigroup to be a (i) principal ideal semigroup, (ii) regular-semigroup.

\subsection{THEOREM. A principal ideal semigroup $\mathrm{S}$ is a multiplication} semigroup if and only if $S$ contains identity.

Proof. Let $S$ be a multiplication semigroup such that $S=x U x S$ for some $x \in S$. By (i) of Theorem 2.14, $s=s^{2}$ so that $s=e s$ for some idempotent element $e \in S$ where $e=x$ or $x s$ for some $s \in S$. Clearly $e$ acts as an identity in $\mathrm{S}$.

Conversely, let $A$ and $B$ be any two ideals of $S$ with $A \subseteq B$. Then $A=a S$ and $B=b S$ for some $a, b \in S$ where $a=b c$ for some c $E S$. Setting $C=C S$ we have $A=B C$, which shows that $S$ is a multiplication semigroup.

2.18 REMARK. We note that there are multiplication semigroups which are neither principal ideal semigroups nor regular semigroups. To see this, we consider $s=\left\{1, x, x^{2}, \ldots, e, f, e f\right\}$ with $\operatorname{ex}=x=f x=e f x$, $e=e^{2}, f=f^{2}$. It can be easily verified that $s$ is a multiplication 
semigroup. However, $S$ is not regular since $x$ is not a regular element and $S$ is not a principal ideal semigroup since the maximal ideal eS $U \mathrm{fS}$ is not a principal ideal.

We shall now determine the conditions under which multiplication semigroups are regular or principal ideal semigroups.

First we obtain conditions for noetherian multiplication semigroups to be regular. In fact, with a weaker hypothesis than noetherian, we have the desired result. However the question is open in arbitrary case.

2.19 THEOREM. Let $S$ be a multiplication semigroup in which every ideal is a product of primary ideals. Then $S$ is regular if and only if every prime ideal is idempotent.

Proof. By (iii) of Theorem 2.14, every prime ideal is idempotent in a regular semigroup.

Conversely, for any $a \in S$ we can write $a S=\prod_{i=1}^{n} Q_{i}$ where each $Q_{i}$ is a primary ideal. By (vii) of Theorem 2.16 for every $i$ we have $Q_{i}=P_{i}{ }_{i}$ for some natural number $n_{i}$ where $P_{i}=\sqrt{Q_{i}}$. Since every $P_{i}$ is an idempotent prime ideal we have then $Q_{i}=P_{i}$ and aS $=\prod_{i=1}^{n} P_{i}=\prod_{i=1}^{n} P_{i}^{2}=a^{2} S$, i.e., $a \in a^{2} S$. Thus $s$ is a regular semigroup.

2.20 COROLLARY. A noetherian multiplication semigroup $S$ is a regular semigroup if and only if every prime ideal is idempotent.

Proof. We show that every ideal is a product of primary ideals in $S$ so that proof follows immediately from the above theorem. If $A$ is any ideal of $S$ then invview of (vi) of Theorem 2.14 we can write 
$A=\bigcap_{i=1}^{n} Q_{i}$ where each $Q_{i}$ is a primary ideal of $s$. By (vii) of Theorem 2.16 we have $Q_{i}=P_{i}{ }^{r}$ for some natural number $r_{i}$, where $P_{i}=\sqrt{Q_{i}}$ is a prime ideal for every $i$. Thus $A=\bigcap_{i=1}^{n} P_{i}{ }^{r}$. We may assume that $P_{i}{ }^{\prime}$ s are distinct. In view of (xviii) of Theorem 2.16 , it then follows that $A=\prod_{i=1}^{n} P_{i}^{r}$ where each $P_{i}{ }^{r}$ is a primary ideal.

2.21 THEOREM. Let $S$ be a multiplication semigroup satisfying any one of the following conditions.

(i) S contains no idempotent prime ideals.

(ii) Every idempotent prime ideal of $S$ contains at most a finite number of prime ideals properly.

(iii) No idempotent prime ideal is equal to the union of all the prime ideals properly contained in it.

(iv) Every idempotent prime ideal of $S$ is finitely generated. Then $S$ is a principal ideal semigroup if and only if $S$ contains identity and the set of prime ideals of $S$ is linearly ordered.

Proof. If $S$ is a principal ideal semigroup then the set of prime ideals of $S$ is linearly ordered in view of (iv) of Theorem 2.14. Now if $S=x \cup \times S$ then $S$ contains an idempotent since $s=s^{2}$ by (i) of Theorem 2.16 and this idempotent is actually an identity element in S.

Conversely, if the set of prime ideals of $S$ is linearly ordered, then every ideal has only one minimal prime divisor so that every ideal is some power of its unique minimal prime divisor. Thus in order to show that $S$ is a principal ideal semigroup it suffices to prove that 
every prime ideal is principal. In fact we need only to prove that every idempotent prime ideal is principal in view of (xvii) of Theorem 2.16.

Now if $S$ satisfies (i), clearly $S$ is a principdil ideal semigroup.

Let $S$ satisfy (ii). Let $p$ be an idempotent prime ideal. If $P$ does not contain any prime ideal properly then for any $x \in P, P$ is the only minimal prime divisor of $x S$ so that $i S=P^{r}=P$. Suppose P contains prime ideals properly. If $Q_{1}, \ldots, Q_{n}$ are prime ideals with each $Q_{i}<P$ then $\underset{i=1}{U} Q_{i}<P$, since $Q_{i}$ 's are linearly ordered. For any $x \in P \backslash \underset{i=1}{\mathrm{n}} Q_{i}, \quad P$ is the only minimal prime divisor of $x S$ so that $\mathbf{x S}=\mathbf{P}$.

If $S$ satisfies (iii), the result follows by a similar argument as in (ii).

Finally, we show that (iv) implies (iii) which completes the proof. If ${ }^{r} \mathrm{P}$ is a finitely generated idempotent prime ideal then in view of (viii) of Theorem 2.14 we can write $p=\bigcup_{i=1} e_{i} S$ where each $e_{i}$ is an idempotent element. Let $\left\{Q_{\alpha}\right\}$ be the set of all prime ideals with each $Q_{\alpha}<$ P. If $U_{\alpha} Q_{\alpha}=P$ then each $e_{i} \in Q_{\alpha_{i}}$ for some $\alpha_{i}$, so that $\mathrm{P}=\bigcup_{i=1}^{\mathrm{n}} \mathrm{e}_{i} \mathrm{~s} \subseteq \bigcup_{i=1}^{\mathrm{n}} \mathrm{Q}_{\alpha_{i}}<\mathrm{P}$, which is impossible. Therefore, $\mathrm{P} \neq \mathrm{U}_{\alpha}$. 2.22 REMARK. If $S$ does not satisfy either of (i), (ii), (iii), (iv) the above theorem need not be true. For example, $s=\left\{1, x_{1}, x_{2}, \ldots\right\}$ with $x_{i} x_{j}=x_{\min }(i, j)$ is a multiplication semigroup containing identity in which the set of prime ideals is linearly ordered. $S$ does not satisfy any one of the conditions $(i)=$ (iv) of the above theorem. Clearly $S$ is not a principal ideal semigroup since the maximalideal 


\begin{abstract}
$\bigcup_{i=1}^{\infty} x_{i} s$ is not principal.
2.23 COROLLARY. A noetherian multiplication semigroup $S$ is a principal ideal semigroup if and only if $S$ contains identity and the set of prime ideals of $S$ is linearly ordered.
\end{abstract}

\title{
2.5 COMMUTATIVE FINITE-DIMENSIONAL SEMIGROUPS
}

In the previous section we obtained conditions for a noetherian multiplication semigroup to be a principal ideal semigroup. We observe that the conditions of being 'noetherian', and 'finite-dimensional' are quite independent. In view of this we study in this section the structure of finite-dimensional multiplication semigroups and discuss when they are principal ideal semigroups. Incidentally, this covers the study of many important multiplication semigroups in the nonnoetherian case.

2.24 THEOREM. For a finite-dimensional multiplication semigroup $\mathrm{S}$, the following are equivalent.

(i) Every idempotent prime ideal is principal.

(ii) $S$ contains identity and the set of prime ideals of $S$ is linearly ordered.

(iii) $S$ is a principal ideal semigroup with identity. Proof. $(i) \Rightarrow$ (ii). Since $s=x \cup \times S$ for some $x \in S$, as in Theorem 2.21 it follows that $S$ contains identity. Let $\operatorname{dim} S=n$. Then there is'a 'chainiof prime ideals $\mathrm{P}_{0}<\mathrm{P}_{1}<\ldots<\mathrm{P}_{\mathrm{n}}$ where $\mathrm{P}_{\mathrm{n}}$ is a maximal ideal by $(x \times i)$ of Theorem 2.16 and $P_{0}$ does not contain 
any prime ideals properly and there are no prime ideals between $P_{i}$ and $P_{i-1}$ for $1 \leq i \leq n$. If $P_{i}=P_{i}^{2}$ for some $i \geq 1$, then by hypothesis $P_{i}$ is principal. In view of (xvi) of Theorem 2.16 , evidently $P_{i-1}$ is the only prime ideal properly contained in $P_{i}$ with no prime ideals between $P_{i}$ and $P_{i-1}$. On the otherhand if $P_{i} \neq P_{i}^{2}$ then by $(x v)$ of Theorem 2.16, $P_{i-1}$ is the only prime ideal properly contained in $P_{i}$ with no prime ideals between $P_{i}$ and $P_{i-1}$. Thus the only prime ideals in $S$ are the $P_{i}$ 's of the above chain, and hence (ii) is evident. $(-i i) \Rightarrow$ (iii). Every idempotent prime ideal contains at most a finite number of prime ideals since $\operatorname{dim} S<\infty$ and since the set of prime ideals of $S$ is linearly ordered. Now from Theorem 2.21, the result follows.

$$
\text { (iii) } \Rightarrow(i) \text { Evident. }
$$

2.25 REMARK. The above theonem is not necessarily true in general. For, consider $s=\left\{1, x_{1}, x_{2}, \ldots\right\}$ with $x_{i} x_{j}=x_{\min (i, j)}$ Clearly $\operatorname{dim} S=\infty$ and the set of prime ideals of $S$ is linearly ordered. The maximal ideal $\bigcup_{i=1}^{\infty} x_{i} S$ is an idempotent prime ideal which is not principal. Thus (i) and (iii) of the above theorem are not true while (ii) is true for this semigroup.

One may feel that noetherian semigroups must be finite-dimensional or vice versa. We claim that it is not so. $s=\left\{1=x_{1}, x_{2}, \ldots\right\}$ with $x_{i} x_{j}=x_{\max }(i, j)$ is a noetherian semigroup which is not finitedimensional and $s=\left\{0,1, x_{1}, x_{2}, \ldots\right\}$ with $x_{i} x_{j}=0$ for all $i, j \geq 1$ is a zero-dimensional semigroup which is not noetherian. In view of this observation we feel that separate status should be given to noetherian 
semigroups and finite-dimensional semigroups in our discussion. In fact this has already been reflected in our discussion.

Now we shall describe the finite-dimensional multiplication semigroups $S$ with $S$ principally generated as an ideal.

2.26 THEOREM. Let $S$ be a finite-dimensional semigroup with $S=x \cup \times S$ for some $x \in S$. Then $S$ is a multiplication semigroup if and only if $S$ contains identity and $S$ is any one of the following types.

(i) $\mathrm{S}$ is a principal ideal semigroup.

(ii) There exists a non-principal idempotent prime ideal $P$ which is a multiplication subsemigroup of $S$ such that every ideal of $S$ not contained in $P$ is principal.

Proof. As in Theorem 2.21, S contains identity. If $S$ contains no idempotent prime ideals then the only idempotent prime ideal is $S$, which is principal. Now suppose $\mathrm{S}$ contains idempotent prime ideals which are all principal. In either of the above two cases $S$ is a principal ideal semigroup by Theorem 2.24 .

Suppose there exists an idempotent prime ideal in $S$ which is not principal. Let $\left\{\mathrm{P}_{\alpha}\right\}$ be the set of all idempotent prime ideals each of which is not principal. Then $U_{\alpha} \mathrm{P}_{\alpha}=\mathrm{P}$ is an idempotent prime ideal which is not principal by (ix) of Theorem 2.14. Clearly $P$ is a proper prime ideal. By $(x)$ of Theorem 2.16, $P$ is a multiplication subsemigroup of $S$. We now claim that the set of prime ideals of $S$ which are not contained in $\mathrm{P}$ is linearly ordered. If $\mathrm{P}$ is the unique maximal ideal of $\mathrm{S}$, the result is trivial. Thus we may assume $\mathrm{P}$ is not maximal. Since dim $S<\infty$, we have a chain of prime ideals $P=Q_{0}<Q_{1}<\ldots<Q_{t}$ 
(where $Q_{t}$ is the unique maximal ideal) with no prime ideals between any two consecutive prime ideals in the above chain. If $Q_{j} \neq Q_{j}^{2}$ for some $j \geq 1$, then $Q_{j-1}$ is the only prime ideal properly contained in $Q_{j}$ with no prime ideals between $Q_{j}$ and $Q_{j-1}$ by (xv) of Theorem 2.16. If $Q_{i}$ is an idempotent prime ideal, then $Q_{i}$ is principal and so by (xvi) of Theorem 2.16 it follows that $Q_{i-1}$ is the only prime ideal properly contained in $Q_{i}$ with no prime ideals between $Q_{i}$ and $Q_{i-1}$. Clearly the prime ideals of $S$ not contained in $P$ are precisely $Q_{1}, \ldots, Q_{t}$. From (xvii) of Theorem 2.16 it follows that every non-idempotent prime ideal, not contained in $P$ is principal. Also every idempotent prime ideal, not contained in $\mathrm{P}$ is principal by the choice of $P$. Clearly every ideal not contained in $P$ has only one minimal prime divisor which is principal. Hence every ideal not contained in $\mathrm{P}$ is some power of a prime ideal and thus is principal. Conversely, a semigroup of type (i) is a multiplication semigroup by Theorem 2.17. Now suppose $S$ is of type (ii). Let $A$ and $B$ be any two ideals of $S$ with $A \subseteq B$. We have the following possibilities: $A, B \subseteq P$ or $A \subseteq P, B \nsubseteq P$ or $A, B \nsubseteq P$. In the first case $A=B C$ for some ideal $C$ of $P$ since $A$ and $B$ are evidently ideals of $P$. Since $\mathrm{C} \subseteq \mathrm{P}$ we have $\mathrm{C}=\mathrm{PD}$ for some ideal $\mathrm{D}$ of $\mathrm{P}$ since $\mathrm{P}$ is a multiplication semigroup. It follows that $C=P D=P^{2} D=P C$. We have then $A=B C P$ where $C P$ is easily seen to be an ideal of $S$. In the second case $B=y S$ for some $y \in S \backslash P$. By definition of $A: B$, clearly $(A: B) B \subseteq A$. If $a \in A$ then $a \in B$ so that $a=y s$ for some $s \in S$ and thus $s \in A: B$. Now $a=y s \in B(A: B)$. Hence $A=(A: B) B$, where $A: B$ is an ideal of $S$. Finally if $A, B \notin P$ then $A=a S$ and 
$B=b S$ for some $a, b \in S \backslash P$ so that $a=b c$ for some $c \in S \backslash P$. Setting $C=c S$ we have $A=B C$. Thus $S$ is a multiplication semigroup.

2.27 REMARK. In general Theorem 2.26 is not necessarily true for any finite-dimensional semigroup. In fact, even for finitely generated (with more than one generator) finite-dimensional semigroups, Theorem 2.26 is not necessarily true. This can be seen by considering $S=\{0, e, f\}$ with $e=e^{2}, f=f^{2}$ and $e f=0$. Here $S=e S U f S$ is a multiplication semigroup which is neither of type (i) nor of type (ii) mentioned in Theorem 2.26 .

2.28 THEOREM. Suppose in a semigroup $\mathrm{S}$, prime ideals are maximal. Let $S=x U \times S$ for some $x \in S$. Then the following are equivalent.

(i) S is a multiplication semigroup.

(ii) $\mathrm{S}$ contains identity, every ideal with prime radical is primairy and every primary ideal is some power ofits radical.

(iii) S contains identity and every proper ideal of $S$ is some power of the unique maximal ideal of $S$.

Proof. (i) $\Rightarrow$ (ii) Noting that $\operatorname{dim} S=0$ the result follows from Theorem 2.26 and (v), (vii) of Theorem 2.16.

$$
\text { (ii) } \Rightarrow \text { (iii) } S \text { contains a unique maximal ideal } M \text { by (i) of }
$$

Theorem 2.14. Now every proper ideal of $S$ has a unique minimal prime divisor $M$ so that the radical of every proper ideal is $M$ and hence every proper ideal of $S$ is some power of $M$.

$$
\text { (iii) } \Rightarrow \text { (i) Trivial. }
$$




\subsection{SOME ADDITIONAL CHARACTERIZATIONS OF COMMUTATIVE MULTIPLICATION SEMIGROUPS}

We now examine whether multiplication semigroups can be completely described with the help of some other ideal-theoretic properties.

2.29 PROPOSITION. Let $S$ be a semigroup in which every ideal has a finite number of distinct minimal prime divisors. Let $S$ satisfy the following properties: (i) $s=s^{2}$, (ii) Every primary ideal is some power of its radical, (iii) $A=\operatorname{Ker} A$ for every ideal $A$ of $S$. Then $S$ is a multiplication semigroup.

Proof. Let $A$ and $B$ be any two ideals of $S$ with $A \subseteq B$. Let $\mathrm{P}_{1}, \mathrm{P}_{2}, \ldots, \mathrm{P}_{\mathrm{n}}$ be the distinct minimal prime divisors of both $\mathrm{A}$ and $B, Q_{1}, Q_{2}, \ldots, Q_{m}$ be the distinct minimal prime divisors of $A$ and not of $B, R_{1}, R_{2}, \ldots, R_{\ell}$ be the distinct minimal prime divisors of $B$ and not of A. In view of (xiii) and (xii) of Theorem 2.14, we may write $A=\bigcap_{i=1}^{n} P_{i}^{r_{i}} \cap \bigcap_{j=1}^{m} Q_{j}^{r_{j}}$ and $B=\bigcap_{i=1}^{n} P_{i}^{t_{i}} \cap \bigcap_{k=1}^{l} R_{k}^{t}$ where $P_{i}^{r_{i} s}$ and $Q_{j}^{r_{j}}$ 's are respectively the isolated $P_{i}$ - and $Q_{j}$ - primary components of $A$ while $P_{i}^{t_{i}}$, and $R_{k}^{t_{k}}$ is are respectively the isolated $P_{i}$ - and $R_{k}$ - primary components of $B$. Since $A \subseteq B$ we have $A \subseteq P_{i}{ }_{i}$ for every $i$. Now

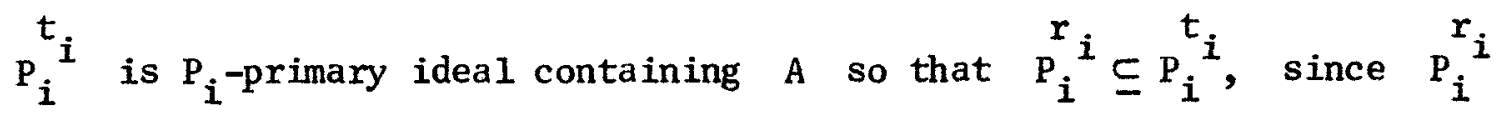
is the isolated $P_{i}$-primary component of $A$. Thus $r_{i} \geq t_{i}$ for every $i$. We set $c=\bigcap_{i=1}^{n} P_{i}^{r_{i}-t} \cap \bigcap_{j=1}^{m} Q_{j}^{r}$. We now claim that $P_{i}^{\prime}$ s and $Q_{j}{ }^{\prime} s$ are precisely the minimal prime divisors of the ideal $B C G_{i}$ suppose $P_{i}\left(Q_{j}\right)$ 
is not minimal prime divisor of $B C$. Then $P_{i}\left(Q_{j}\right)$ properly contains a minimal prime divisor $\mathrm{L}$ of $\mathrm{BC}$. Now

$\prod_{i=1}^{n} P_{i}{ }^{r} \prod_{k=1}^{\ell} R_{k}{ }^{t} \prod_{j=1}^{m} Q_{j}^{r}=\prod_{i=1}^{n} P_{i}{ }^{t} \prod_{k=1}^{\ell} R_{k}{ }^{t} \prod_{i=1}^{n} P_{i}{ }^{r_{i}-t_{i}} \prod_{j=1}^{m} Q_{j}{ }^{r_{j}}$

$\subseteq B C \subseteq L<P_{i}\left(Q_{j}\right)$. Clearly for some $t, P_{t}$ or $R_{t}$ or $Q_{t} \subseteq L<P_{i}\left(Q_{j}\right)$, which is not possible (we note that every $R_{k}$ contains some $Q_{j}$ properly). Thus $P_{j}$ 's and $Q_{j}$ 's are minimal prime divisors of $B C$. Now we show that these are precisely the minimal prime divisors of $\mathrm{BC}$. Let $\mathrm{L}$ be a minimal prime divisor of $\mathrm{BC}$. Then $\mathrm{L}$ contains $\mathrm{B}$ or $\mathrm{C}$. We have $\mathrm{A} \subseteq \mathrm{C}$. so that $A \subseteq L$. Now $L$ contains a minimal prime divisor of $A$ so $L$ contains $P_{i}$ or $Q_{j}$ for some $i, j$. But $P_{i}{ }^{\prime} s, Q_{j}^{\prime}$ s are minimal prime divisors of $\mathrm{BC}$ so $\mathrm{BC} \subseteq \mathrm{P}_{i} \subseteq \mathrm{L}$ or $\mathrm{BC} \subseteq \mathrm{Q} \subseteq \mathrm{L}$. This shows that $L=P_{i}$ or $Q_{j}$. Now we can write $B C=\bigcap_{i=1}^{n} P_{i}^{s_{i}} \cap \bigcap_{j=1}^{m} Q_{j}^{s}$ as before, since $B C=\operatorname{Ker} B C$. Here $P_{i}^{s^{i}}, s$ and $Q_{j}^{s^{j} s}$ are respectively the isolated $P_{i}$ - and $Q_{j}$ - primary componentscof $B C$. We have

$\prod_{i=1}^{n} P_{i}{ }^{r} \prod_{k=1}^{n} R_{k}{ }^{t} \prod_{j=1}^{m} Q_{j}{ }^{r} \subseteq B C \subseteq P_{i}{ }^{i}, Q_{j}{ }^{s}$ for every $i$ and $j$. clearly $P_{i}^{{ }^{r}} \subseteq P_{i}^{s_{i}}$ and $Q_{j}^{{ }^{j}} \subseteq Q_{j}^{{ }^{j}}$ for every $i$ and $j$ since $P_{i}^{{ }^{i}}{ } s$ and $Q_{j}^{{ }_{j}} j_{i}$ are respectively $P_{i}$-primary and $Q_{j}$-primary ideals. Thus $A \subseteq B C$. Trivially $B C \subseteq P_{i}{ }^{r_{i}}$ and $Q_{j}{ }^{r_{j}}$ for every $i$ and $j$ so that $\mathrm{BC} \subseteq \mathrm{A}$. Hence $\mathrm{A}=\mathrm{BC}$ which shows that $\mathrm{S}$ is a multiplication semigroup.

2.30 THEOREM. Let $S$ be a semigroup satisfying any one of the 
following conditions.

(a) Every ideal of $S$ has only a finite number of distinct minimal prime divisors and is a product of its minimal prime divisors.

(b) $\mathrm{S}$ is noetherian.

(c) Every ideal of $S$ has only a finite number of distinct minimal prime divisors and $\mathrm{dim} \mathrm{s}^{1} \leq 1$.

(d) Every ideal of $\mathrm{S}$ has only a finite number of distinct minimal prime divisors and prime ideals of $S$ are maximal. Then $S$ is a multiplication semigroup if and only if $S$ satisfies the conditions (i), (ii) and (iii) of Proposition 2.29.

Proof. In view of (i) and (vii) of Theorem 2.16, we only have to show that $A=\operatorname{Ker} A$ for every ideal $A$ of $S$ if $S$ is a multiplication semigroup satisfying any one of the conditions (a) - (d).

Let $S$ satisfy (a). For any ideal $A$ of $s$, let $P_{1}, P_{2}, \ldots, P_{n}$ be the distinct minimal prime divisors of A. In view of (v), (vii) of Theorem 2.16 and (xii) of Theorem 2.14, we may choose $k_{i} \quad(1 \leq i \leq n)$ such that $P_{i}{ }_{i}$ is the isolated $P_{i}$-primary component of $A$. Now we can write Ker $A=\bigcap_{i=1}^{n} P_{i}^{k}$. But by (xviii) of Theorem 2.16, we have $\bigcap_{i=1}^{n} P_{i}^{k_{i}}=\prod_{i=1}^{n} P_{i}^{k_{i}}$. By hypothesis $A=\prod_{i=1}^{n} P_{i}^{b}$ for some natural numbers $\ell_{i}$. Thus $A=\prod_{i=1}^{n} P_{i}{ }_{i}^{i_{i}} \subseteq \operatorname{Ker} A=\prod_{i=1}^{n} P_{i}{ }^{k}$. By $(v)$ of Theorem 2.16, every $P_{i}^{k_{i}}$ is $P_{i}$-primary and so $P_{i}^{{ }^{l}} \subseteq P_{i}^{k_{i}}$ for every $i$ since $P_{j} \nsubseteq P_{i}$ for every $j \neq i$. Now $A \subseteq P_{i}^{l}$ and $P_{i}^{k}$ is isolated $P_{i}$-primary component of $A$, and so $P_{i}^{{ }^{k}} \subseteq P_{i}^{l}$, since $P_{i}^{l}$ is $P_{i}$-primary 
ideal containing $A$ by $(v)$ of Theorem 2.16. Thus for every $i$ we have $\mathrm{P}_{i}^{k_{i}}=\mathrm{P}_{i}^{l_{i}}$ and hence $\mathrm{A}=\operatorname{Ker} \mathrm{A}$.

If $S$ satisfies (b) then in view of $(x x)$ of Theorem 2.16, we have $A=\operatorname{Ker} A$ for every ideal $A$ of $S$.

If $S$ satisfies (c) or (d) then $A=\operatorname{Ker} A$ for every ideal $A$ in view of (xxii) of Theorem 2.16 .

We note that if $S$ satisfies (b) then every ideal of $S$ has a finite number of distinct minimal prime divisors in view of (vii) of Theorem 2.14. Now proof is complete in view of the above proposition.

\subsection{COMMUTATIVE CHAINED SEMIGROUPS}

It is well known that every monoid is embeddable in a chained monoid in the cancellative case. There are non-cancellative monoids which are embeddable in chained monoids (as total quotient monoids). Now we shall investigate how the multiplication property can be transferred from a monoid to its total quotient monoid and conversely.

2.31 DEFINITION. A chained semigroup is a semigroup in which the set of ideals is linearly ordered by set-inclusion.

In a regular semigroup $s$, if the set of prime ideals is linearly ordered, then the idempotents of $S$ form a chain. It then follows from Theorem 2.4 of [ 14$]$ that a regular semigroup is chained if and only if the set of all its prime ideals is linearly ordered. It is interesting to note that this result can be generalized to multiplication semigroups. However, in general this is not the case. For example, 
$S=\left\{1, a, a^{2}, e\right\}$ with $e=e^{2}$, ea $=a^{2}=a^{3}=e a^{2}$ is a semigroup in which $\left\{a, a^{2}\right\},\left\{e, a, a^{2}\right\}$ and $s$ are the only prime ideals. The set of prime ideals is clearly linearly ordered, but the ideals $\left\{e, a^{2}\right\}$ and $\left\{a, a^{2}\right\}$ are not comparable.

2.32 THEOREM. A multiplication semigroup $S$ is a chained semigroup if and only if the set of prime ideals of $S$ is linearly ordered.

Proof. Suppose the set of prime ideals of $S$ is linearly ordered. Let $A$ and $B$ be any two ideals of $S$. If $P$ is a minimal prime divisor of both Aland $B$ then $P$ is the only minimal prime divisor of both $A$ and $B$. Now $A=P^{m}$ and $B=P^{n}$ for some natural numbers $m$ and $n$. It follows that $A \subseteq B$ or $B \subseteq A$ according as in $\geq n$ or $m \leq n$. Now suppose $P$ and $Q(\neq P)$ are minimal prime divisors of $A$ and $B$ respectively. Then $A=P^{m}$ and $B=Q^{n}$ for some natural numbers $\mathrm{m}$ and $\mathrm{n}$ ssince $\mathrm{p}$ and $\mathrm{Q}$ are respectively the only minimal prime divisors of $A$ and $B$. If $P<Q$ then $P=P Q=\ldots=P Q^{n}$ by (iii) of Theorem 2.16 so that $\mathrm{P}^{\mathrm{m}}=\mathrm{P}^{\mathrm{m}} \mathrm{Q}^{\mathrm{n}}$, i.e., $A=A B \subseteq B$. If $\mathrm{Q}<\mathrm{P}$ then it can be verified in a similar way that $\mathrm{B} \subseteq \mathrm{A}$. Thus the set of ideals of $S$ is linearly ordered. Hence $S$ is chained.

The other implication is evident.

We now characterize chained multiplication semigroups.

2.33 THEOREM. A semigroup $S$ is a chained multiplication semigroup if and only if (i) $\mathrm{s}=\mathrm{s}^{2}$, (ii) every ideal of $\mathrm{s}$ is primary, (iii) every ideal has a unique minimal prime divisor and is some power of its unique minimal prime divisor. 
Proof. Let $S$ be a chained multiplication semigroup. Then (i) is evident by ( $i$ ) of Theorem 2.16. Now if $A$ is any ideal of $S$ then $A$ has a unique minimal prime divisor $P$, since $S$ is chained. Clearly $\sqrt{A}=P$ and so by $(v)$ and (vii) of Theorem 2.16, $A=P^{n}$ for some natural number $n$.

To prove the converse we first note that $A=A S$ for every ideal $A$ of $S$. For, if $S$ is a minimal prime divisor of $A$ then $A=S^{n}=S$ and so $A=A S$ since $S=S^{2}$. Suppose $P$ is a proper minimal prime divisor of A. We claim that $P$ is also a minimal prime divisor of AS. For otherwise by (ii) of Theorem 2.14, P contains a minimal prime divisor $Q(\neq S)$ of $A S$ since $A S \subseteq A \subseteq P$. Now $A S \subseteq Q<P$ and so $A \subseteq Q$. But then $A \subseteq Q<P$, which contradicts the fact that $P$ is a minimal prime divisor of A. Thus $\mathrm{P}$ is a minimal prime divisor of AS. Now $A=P^{m}$ and $A S=P^{n}$ for some natural numbers $m$ and $A$. Since every ideal is primary $P^{n}$ is $P$-primary and so $A \subseteq P^{n}$ since $S q P$. Thus $A=P^{m} \subseteq P^{n}=A S \subseteq A$ which shows that $A=A S$ for every ideal $A$ of $S$. Now let $A$ and $B$ be any two ideals of $S$ with $A \subset B$. In view of the above observation it suffices to consider the case when $A<B$ with $B \neq S$. If $P$ is a minimal prime divisor of both $A$ and $B$ then $A=P^{m}$ and $B=p^{n}$ for some natural numbers $m$ and $n$ where $\mathrm{m}>\mathrm{n}$. Setting $\mathrm{C}=\mathrm{P}^{\mathrm{m}-\mathrm{n}}$ we have $\mathrm{A}=\mathrm{BC}$. Now suppose $\mathrm{P}$ and $\mathrm{Q}(\neq \mathrm{P})$ be minimal prime divisors of $A$ and $B$ respectively. Then $A=P^{m}$ and $B=Q^{n}$ for some natural numbers $m$ and $n$. clearly $P<Q$. We note that $P$ is a minimal prime divisor of $\mathrm{P}^{\mathrm{m}} \mathrm{Q}^{\mathrm{n}}$. For otherwise by (ii) of Theorem 2.14, $\mathrm{P}^{\mathrm{m}} \mathrm{Q}^{\mathrm{n}} \subseteq \mathrm{L}<\mathrm{P}$ where $\mathrm{L}$ is a minimal prime divisor of $P^{m} Q^{n}$. This shows that $L$ contains $P$ or $Q$, which is not possible. 
Hence $\mathrm{P}$ is a minimal prime divisor of $\mathrm{p}^{\mathrm{m}} \mathrm{Q}^{\mathrm{n}}$. Then by (iii), $P^{m} Q^{n}=P^{k}$ for some natural number $k$. Since $P^{k}$ is $P$-primary, $\mathrm{P}^{\mathrm{m}} \subseteq \mathrm{p}^{\mathrm{k}}$ since $\mathrm{Q}^{\mathrm{n}} \nsubseteq \mathrm{P}$. Thus $\mathrm{P}^{\mathrm{m}} \subseteq \mathrm{P}^{\mathrm{k}}=\mathrm{P}^{\mathrm{m}} \mathrm{Q}^{\mathrm{n}} \subseteq \mathrm{P}^{\mathrm{m}}$ which shows that $A B=P^{m} Q^{n}=P^{k}=P^{m}=A$. Hence $S$ is a multiplication semigroup. Now we show that $S$ is a chained semigroup. For this it suffices to show that the set of prime ideals of $S$ is linearly ordered, because of Theorem 2.32. Let $P$ and $Q$ be any two prime ideals of $S$. Since $\mathrm{PQ} \subseteq \mathrm{P}, \quad \mathrm{P}$ contains a minimal prime divisor $\mathrm{L}$ of $\mathrm{PQ}$ by (ii) of Theorem 2.14. Hence $\mathrm{PQ} \subseteq \mathrm{L} \subseteq \mathrm{P}$. Also $\mathrm{Q} \subseteq \mathrm{L} \subseteq \mathrm{P}$ or $\mathrm{P} \subseteq \mathrm{L} \subseteq \mathrm{P}$. Thus $\mathrm{Q} \subseteq \mathrm{P}$ or $\mathrm{L}=\mathrm{P}$ is a minimal prime divisor of $\mathrm{PQ}$. If $\mathrm{Q} \nsubseteq \mathrm{P}$ then $P$ is the only minimal prime divisor of $P Q$ and so $P Q=P^{r}$ for some natural number $r$. Thus $P^{r}=P Q \subseteq Q$ which shows that $P \subseteq Q$. In the following $(S, M)$ denotes a commutative monoid with a unique maximal ideal $M$.

2.34 THEOREM. Let $(S, M)$ be a monoid with $T$ as its total quotient monoid. Then the following are equivalent.

(i) $\mathrm{S}$ is a chained multiplication semigroup.

(ii) Every ideal of $S$ is some power of a prime ideal and whenever $P$ and $Q$ are any two prime ideals of $S$ with $P<Q$ then $P=P Q$.

(iii) $S$ is a multiplication semigroup such that the set of prime ideals of $\mathrm{T}$ is linearly ordered.

(iv) Every ideal of $S$ has a unique minimal prime divisor and is some power of its unique minimal prime divisor; every ideal is primary.

Proof. (i) $\Rightarrow$ (ii) follows from Theorem 2.33 and (iii) of 
Theorem 2.16.

(ii) $\Rightarrow$ (iii). Let $A$ and $B$ be any two ideals of $S$ with $A \subseteq B$. If $A=\mathrm{P}^{r}$ and $B=\mathrm{P}^{S}$ for some prime ideal $\mathrm{P}$ and for some natural numbers $r$ and $s$, then clearly $r \geq s$ so that $A=B C$ where $c=\mathrm{P}^{\mathrm{r}-\mathrm{s}}$ or $\mathrm{s}$ ix according as $\mathrm{r}>\mathrm{s}$ or $\mathrm{r}=\mathrm{s}$. Now suppose $\mathrm{A}=\mathrm{P}^{\mathrm{r}}$ and $B=Q^{S}$ for some natural numbers $r$ and $s$, where $P$ and $Q$ are two distinct prime ideals. Clearly $P<Q$ so that $P=P Q=\ldots=P Q^{S}$. Thus $P^{r} Q^{s}=P^{r}$, i.e., $A B=A$. Hence $S$ is a multiplication semigroup. Finally, if $P^{*}$ and $Q^{*}$ are any two prime ideals of $T$ we show that $\mathrm{P}^{*} \subseteq \mathrm{Q}^{*}$ or $\mathrm{Q}^{*} \subseteq \mathrm{P}$. Clearly $\mathrm{P}^{*} \cap \mathrm{S}$ and $\mathrm{Q}^{*} \cap \mathrm{S}$ are ideals of $\mathrm{S}$. In fact, they are prime ideals of $s$. For, let $x, y \in S$ with $x y \in P^{*} \cap S$. Suppose that $x \notin P^{*} \cap S$. Then $x \notin P^{*}$. But $P^{*}$ is a prime ideal of $T$ and so $y \in P^{*}$ which shows that $y \in P^{*} \cap S$. Thus $P^{*} \cap S$ and similarly $Q * \cap S$ are prime ideals of $S$ which may be denoted by $P_{1}$ and $Q_{1}$. We may write $P_{1} Q_{1}=L^{k}$ for some natura 1 number $k$ and for some prime ideal $L$ of $S$. Now $P_{1}$ or $Q_{1} \subseteq L$. Since $L^{\mathrm{k}} \subseteq \mathrm{P}_{1} \cap \mathrm{Q}_{1}$ we have $\mathrm{L}=\mathrm{P}_{1}$ or $\mathrm{Q}_{1}$. Thus $\mathrm{P}_{1} \mathrm{Q}_{1}=\mathrm{P}_{1}^{\mathrm{k}}$ or $\mathrm{Q}_{1}^{\mathrm{k}}$. If $\mathrm{P}_{1} \mathrm{Q}_{1}=\mathrm{P}_{1}^{\mathrm{k}}$ then $\mathrm{P}_{1}^{\mathrm{k}} \subseteq \mathrm{Q}_{1}$ and thus $\mathrm{P}_{1} \subseteq \mathrm{Q}_{1} \cdot$ Similarly, if $\mathrm{P}_{1} \mathrm{Q}_{1}=\mathrm{Q}_{1}^{\mathrm{k}}$ then $\mathrm{Q}_{1} \subseteq \mathrm{P}_{1}$. Now we claim that $\mathrm{P}^{*}=\left(\mathrm{P}^{*} \cap \mathrm{S}\right) \mathrm{T}$ and $Q^{*}=\left(Q^{*} \cap \mathrm{S}\right) \mathrm{T}$. Let $\mathrm{u} / \mathrm{v} \in \mathrm{P}^{*}$. We may write $\mathrm{u} / \mathrm{v}=\mathrm{u} / 1 \cdot 1 / \mathrm{v}$ where $\mathrm{u} / \mathrm{l}=\mathrm{u} / \mathrm{v} \ldots \mathrm{v} / \mathrm{l} \in \mathrm{P} * \mathrm{~T} \subseteq \mathrm{P}^{*}$ and $\mathrm{u} / \mathrm{l}$ when identified with $\mathrm{u}$, is in $\mathrm{s}$. Thus $u / l \in P^{*} \cap S$ and $1 / v \in T$. Hence $u / v \in\left(P^{*} \cap S\right) T$. Evidently $\left(\mathrm{P}^{*} \cap \mathrm{S}\right) \mathrm{T} \subseteq \mathrm{P}^{*} \mathrm{~T} \subseteq \mathrm{P}^{*}$. Thus $\left(\mathrm{P}^{*} \cap \mathrm{S}\right) \mathrm{T}=\mathrm{P}^{*}$ and $\left(\mathrm{Q}^{*} \cap \mathrm{S}\right) \mathrm{T}=\mathrm{Q}^{*}$. From the above we have $P^{*} \cap S \subseteq Q^{*} \cap S$ or $Q^{*} \cap S \subseteq P^{*} \cap S$. It follows that $\left(P^{*} \cap \mathrm{S}\right) \mathrm{T} \subseteq\left(\mathrm{Q}^{*} \cap \mathrm{S}\right) \mathrm{T}$ or $\left(\mathrm{Q}^{*} \cap \mathrm{S}\right) \mathrm{T} \subseteq\left(\mathrm{P}^{*} \cap \mathrm{S}\right) \mathrm{T}$, i.e., $\mathrm{P}^{*} \subseteq \mathrm{Q}^{*}$ or 
$\mathrm{Q}^{*} \subseteq \mathrm{P}^{*}$. Thus prime ideals of $\mathrm{T}$ are linearly ordered.

$$
\text { (iii) } \Rightarrow \text { (iv). For any proper ideal } A \text { of } S \text { if } M \text { is a minimal }
$$

prime divisor of $A$, then $M$ is the only minimal prime divisor of $A$ and so $A=M^{r}$ for some natural number $r$ by $(v)$ and (vii) of Theorem 2.16. Suppose $P(\neq M \varphi)$ and $Q(\neq M)$ be any two distinct minimal prime divisors of $A$. Now we show that $P T$ is a prime ideal of $T$. Evidently $\mathrm{PT}$ is an ideal of $\mathrm{T}$. Let $u_{1} / v_{1}, u_{2} / v_{2} \in \mathrm{T}$ be such that $\mathrm{u}_{1} \mathrm{u}_{2} / \mathrm{v}_{1} \mathrm{v}_{2} \in \mathrm{PT}$. Suppose $\mathrm{u}_{1} / \mathrm{v}_{1} \notin \mathrm{PT}$. Clearly $\mathrm{u}_{1} \notin \mathrm{P}$. Since $\mathrm{u}_{1} \mathrm{u}_{2} / \mathrm{v}_{1} \mathrm{v}_{2} \in \mathrm{PT}$ there exist $\mathrm{x}_{1} \in \mathrm{P}$ and a cancellable element $\mathrm{y}_{1} \in \mathrm{S}$ such that $u_{1} u_{2} / v_{1} v_{2}=x_{1} / y_{1}$. Now $u_{1} u_{2} y_{1}=v_{1} v_{2} x_{1} \in P$ since $x_{1} \in P$, $v_{1} v_{2} \in S$. Now $u_{1} \notin P$ and $y_{1}$ being a cancellable element does not belong to $\mathrm{P}$ in view of (iii) and $(\mathrm{xxix})$ of Theorem 2.16 , so that $\mathrm{u}_{2} \in \mathrm{P}$. Thus $\mathrm{u}_{2} / \mathrm{v}_{2} \in \mathrm{PT}$. By a similar argument $\mathrm{QT}$ is also a prime ideal of $T$. Since the set of prime ideals of $T$ is linearly ordered we have either $\mathrm{PT} \subseteq \mathrm{QT}$ or $\mathrm{QT} \subseteq \mathrm{PT}$. If $\mathrm{PT} \subseteq \mathrm{QT}$ we claim that $\mathrm{P} \subseteq \mathrm{Q}$. Let $x \in P$. Then $x \in P T \subseteq Q T$ so that we may write $x / 1=y / s$ where $y \in Q$ and $s$ is a cancellable element in $S$. Now $x s=y \in Q$. Since $s$ is a cancellable element $s \notin Q$ by a similar argument as before, so that $x \in Q$. Thus $P \subseteq Q$. On the otherhand, if $Q T \subseteq P T$ it follows in a similar way that $Q \subseteq P$. This shows that $P$ and $Q$ cannot both be minimal prime divisors of the ideal A. Thus $A$ has a unique minimal prime divisor and by (v) and (vii) of Theorem 2.16, A is some power of its unique minimal prime divisor. Clearly every ideal is primary.

$$
\text { (iv) } \Rightarrow \text { (i) follows from Theorem } 2.33 \text {. }
$$


2.35 THEOREM. Let $(S, M)$ be a commutative monoid. Then the following are equivalent.

(i) $\mathrm{S}$ is a multiplication semigroup in which every ideal is a unique product of prime ideals.

(ii) $S$ is a principal ideal semigroup in which every ideal is some power of $M$ and powers of $M$ properly descend.

(iii) $M=x S$ for some $x \in S$, powers of $M$ properly descend and every element of $S$ is of the form $x^{x}$ for some non-negative integer $\mathbf{r}$ and for some unit $u$.

Proof. (i) $\Rightarrow$ (ii). If $P$ is a prime ideal such that $P<M$ then $\mathrm{P}=\mathrm{MP}$ by (iii) of Theorem 2.16 which contradicts the unique product. This shows that there are no prime ideals.in $S$ except $M$. Now every ideal is some power of $M$ by $(v)$ and (vii) of Theorem 2.16. Since every ideal is unique product of prime ideals, evidently powers of $M$ properly descend. Now for any $x \in M \backslash M^{2}, x S^{1}=x S=M$. Every ideal, being some power of $M$, is thus principal.

(ii) $\Rightarrow$ (iii). Evident.

$($ iii $) \Rightarrow(i)$. Let $A$ be any proper ideal of $S$. Let $n=\min \left\{m \mid x^{m} \in A\right\}$. We claim that $A=M^{n}$. Clearly $M^{n} \subseteq A$. For any $a \in A$ we have $a=x^{r} u$ where $u$ is a unit, and so $x^{r} \in A$. Clearly $x^{r} \in M^{n}=x^{n} S$ and thus a $\in M^{n}$. Thus $A=M^{n}$. Now, let $A$ and $B$ be any two ideals of $S$ with $A \subseteq B .1$ To show that $S$ is a multiplication semigroup, it suffices to consider the case $A<B$ with $B \neq S$, since we have $A=A S$. From the above $A=M^{n}$ and $B=M^{m}$ for some natural numbers $n$ and $m$ where $n>m$. Setting $C=M^{n-m}$ we have $A=B C$. 
Since every ideal is some power of $M$ where powers of $M$ properly descend, evidently every ideal is a unique product of prime ideals. 


\section{GENERAL DISCUSSION}

In commutative ring theory, multiplication rings have been extensively studied by various people including Gilmer [7], Mott [10]. Multiplication rings have been defined exactly the same way as multiplication semigroups.

We now observe some major differences between the multiplication rings (containing identity) and commutative multiplication semigroups. This incidentally focuses one's attention to the fact that the techniques employed in obtaining the structure theorems of multiplication rings and multiplication semigroups are different.

In a multiplication ring $R$ containing identity we have 't: (i): $\operatorname{dim} \quad R \leq 1$;

(ii) if $M$ is a maximal ideal of $R$ and $P$ is a non-maximal prime ideal of $R$ contained in $M$ then $P=M^{\omega}$;

(iii) every non-maximal prime ideal of $R$ is an idempotent ideal. The above properties are not necessarily true for multiplication semigroups.

(i) We see that the dimension of a commutative multiplication semigroup need not be even finite. This fact is illustrated by the example $S=\left\{1, x_{1}, x_{2}, \ldots\right\}$ with $x_{i} x_{j}=x_{\min (i, j)^{\circ}}$

(ii)' In a commutative multiplication semigroup it is not necessarily true that $P=M^{\omega}$ for any non-maximal prime ideal $P$ contained in a maximal ideal $M$. This can be seen by considering $P=x_{1} S$ in the above example.

(iii) The following example shows that every non-maximal prime 
ideal is not necessarily idempotent in multiplication semigroups. Let $S=s_{1} \cup s_{2} \cup e$ where $s_{1}$ and $s_{2}$ are isomorphic copies of the additive semigroup of positive integers and $e$ is the identity adjoined to $s_{1} \cup s_{2}$. We define multiplication in $s$ as follows:

$$
\begin{array}{rlrl}
a \cdot b & =a+b \quad \text { if } & a, b \in S_{1} \text { or } S_{2}, \\
& =b \quad \text { if } \quad a \in S_{1}, \quad b \in s_{2} .
\end{array}
$$

$\mathrm{S}_{2}$ is a non-maximal prime ideal of $\mathrm{S}$ which is not an idempotent ideal.

Unlike in commutative rings we have nice structure theorems of commutative multiplication semigroups irrespective of whether they contain identity or not: However, as in commutative ring theory we do not have a characterization of commutative multiplication semigroups, in the general case.

In the following we mention some interesting open problems.

(1) Find necessary and sufficient conditions for a semisimple right multiplication-, left multiplication semigroup. to be regular. In 1.27, we constructed an example of a semigroup of the above sort.

(2) We conjecture that every ideal has only a finite number of distinct minimal prime divisors in commutative multiplication semigroups.

If this is proved then we can have a general characterization of commutative multiplication semigroups in terms of some ideal-theoretic properties other than the defining property.

(3) Determine the structure of infinite-dimensional commutative m 
multiplication semigroups. In this context we mention that we covered some portions of this situation in Theorems $2.19,2.30$ and 2.33 .

(4) When do multiplication semigroups admit a ring stmucture?

In general a multiplication semigroup need not admit a ring structure. For example, the direct product $N \times G$, where $N$ is the additive semigroup of non-negative integers and $G$ is a trivial group, does not admit a ring structure.

(5) A multiplication ring need not be a multiplication semigroup. For example, $(\mathrm{z},+, \cdot)$, the ring of integers with usual addition and multiplication operations, is one such.

Find conditions when a multiplication ring can be a multiplication semigroup.

(6) The representation theory for inverse semigroups has been developed by McAlister, Munn, Petrich etc., as noted in [2]. Develop the representation theory for at least commutative multiplication semigroups. This covers incidentally the representation theory of commutative principal ideal semigroups which is a natural class of semigroups for many important considerations.

(7) One may ask whether it is possible to construct larger multiplication semigroups in a natural way by considering the direct products or extensions.

The answer is "no" in general. For example, $\mathbb{Z}_{2}=\{\overline{0}, \overline{1}\}$ and $\mathbb{Z}_{4}=\{\overline{0}, \overline{1}, \overline{2}, \overline{3}\}$ are multiplication semigroups while their direct product $\mathbb{Z}_{2} \times \mathbb{Z}_{4}$ is not, since $\mathbb{Z}_{2} \times 2 \mathbb{Z}_{4} \neq\left[(0) \times \mathbb{Z}_{4} \cup \mathbb{Z}_{2} \times 2 \mathbb{Z}_{4}\right]$ A for any ideal $A$ of $\mathbb{Z}_{2} \times \mathbb{Z}_{4}$.

The semigroup $S=\left\{1, a, a^{2}, e\right\}$ with $e a=a^{2}=a^{3}$ and $e=e^{2}$ is 
an extension of the regular semigroup $\left\{e, a^{2}\right\}$ by a principal ideal monoid $\{\overline{0}, \overline{1}, \bar{a}\}$ and thus $S$ is an extension of a multiplication semigroup by another multiplication semigroup. However, $S$ itself is not a multiplication semigroup since $\left\{a, a^{2}\right\} \neq\left\{e, a, a^{2}\right\}$ A for any ideal $A$ of $S$.

The natural questions in this direction are:

(i) When can 'a direct product of two multiplication semigroups' be a multiplication semigroup?

(ii) When is 'an extension of a multiplication semigroup by a multiplication semigroup' again a multiplication semigroup? 


\section{BIBLIOGRAPHY}

1. Anderson, D. D., Multiplicative Lattices, Ph.D. Thesis (1974), University of Chicago.

2. Clifford, A. H. and G. B. Preston, The algebraic theory of Semigroups, Vol. I, Math, Surveys No. 7, Amer. Math. Soc., Providence, $\overline{1961 .}$

3. The algebraic theory of Semigroups, Vol. II, Math. Surveys No. 7, Amer. Math. Soc., Providence, 1967.

4. Clifford, A. H., The fundamental representation of a regular semigroup, Math. Dept. of Tulane Univ. (July 1974), 65 pp.

5. Dorofeeva, M. P., V. L. Mannepalli and M. Satyanarayana, Prüfer and Dedekind Monoids, Semigroup Forum 9(1975), 294-309.

6. Dorofeeva, M. P. and M. Satyanarayana, Non-cancellative Prüfer Monoids (submitted).

7. Gilmer, R. W. and J. L. Mott, Multiplication rings as rings in which ideals with prime radicals are primary, Trans. Amer. Math. Soc. $\overline{114(1965), 40-52 .}$

8. Grillet, P. A., On regular semigroups and their multiplication,

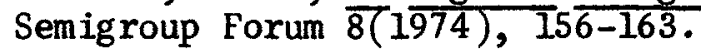

9. Larsen, M. D. and P. J. McCarthy, Multiplicative theory of ideals, Academic Press, New York, 1971.

10. Mott, J. L., Equivalent conditions for a ring to be a multiplication ring, Canad. J. Math. 16(1964), 429 $\overline{-434}$.

11. Namboodripad, K. S. S., On some classes of regular semigroups, Semigroup Forum $2(1971), \overline{2} 6 \overline{4-27} 0$.

12. Petrich, M., Introduction to semigroups, Merrill Publ. Co., Columbus, ohio, 1973.

13. Satyanarayana, M., On semigroups admitting ring structure, Semigroup Forum 3(1971), 43-50.

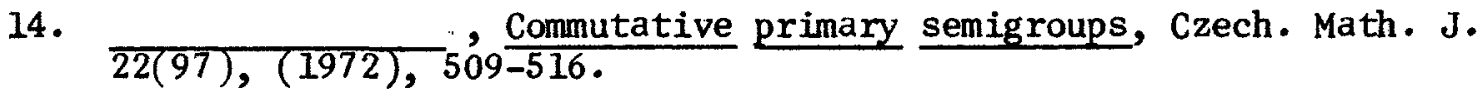

15. $\frac{}{6(1973), 317-329}$, On left cancellative semigroups, Semigroup Forum

16. On a class of semisimple semigroups, Semigroup Forum 10(1975), $129-13 \overline{8}$. 
17., Structure and ideal theory of commutative semigroups (submitted).

18. Schwarz, S., Prime ideals and maximal ideals in semigroups, Czech. Math. J. 19( $(\overline{1969)}, \overline{72-79}$. 